\title{
A Stothers-Mason theorem with a difference radical
}

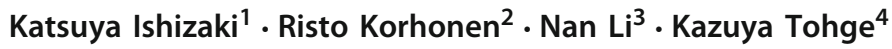

Received: 16 April 2019 / Accepted: 26 July 2020 / Published online: 28 September 2020

(c) The Author(s) 2020

\begin{abstract}
Differential calculus is not a unique way to observe polynomial equations such as $a+b=c$. We propose a way of applying difference calculus to estimate multiplicities of the roots of the polynomials $a, b$ and $c$ satisfying the equation above. Then a difference $a b c$ theorem for polynomials is proved using a new notion of a radical of a polynomial. Results, for example, on the non-existence of polynomial solutions to difference Fermat and difference Super-Fermat functional equations are given as applications. We also introduce a truncated second main theorem for differences, and use it to consider these functional equations with non-polynomial entire solutions. Equations with polynomial or non-polynomial solutions are observed to see the sharpness of results obtained.
\end{abstract}

Mathematics Subject Classification Primary 30D35; Secondary 30C10 · 39A10

The first author was supported by the discretionary budget (2017 and 2018) of the President of the Open University of Japan, and JSPS KAKENHI Grant Number JP20K03658. The second author wishes to acknowledge partial support by the Academy of Finland Grant 286877. The third author was supported in part by the NNSF of China 11801215, the Grant ZR2016AQ20 from the NSF of Shandong Province, and the NNSF of China No. 11371225. The work of the fourth author was supported by JSPS KAKENHI Grant Number JP16K05194.

$\bowtie$ Risto Korhonen risto.korhonen@uef.fi

Katsuya Ishizaki ishizaki@ouj.ac.jp

Nan $\mathrm{Li}$

nanli32787310@163.com

Kazuya Tohge

tohge@se.kanazawa-u.ac.jp

1 The Open University of Japan, 2-11 Wakaba, Mihama-ku, Chiba 261-8586, Japan

2 Department of Physics and Mathematics, University of Eastern Finland, P.O. Box 111, 80101 Joensuu, Finland

3 School of Mathematics, Qilu Normal University, Jinan 250200, Shandong, People's Republic of China

4 Institute of Science and Engineering, Kanazawa University, Kakuma-machi, Kanazawa 920-1192, Japan 


\section{Introduction}

The Stothers-Mason theorem states that if relatively prime polynomials $a, b$ and $c$, not all of them identically zero, satisfy

$$
a+b=c,
$$

then $\operatorname{deg} c \leq \operatorname{deg} \operatorname{rad}(a b c)-1$, where the radical $\operatorname{rad}(a b c)$ is the product of distinct linear factors of $a b c$ [27,37], see also [13,35]. An elementary application of Mason's theorem is that if $x, y$ and $z$ are non-trivial relatively prime polynomials satisfying

$$
x^{n}+y^{n}=z^{n},
$$

where $n \in \mathbb{N}$, then $n \leq 2$. The Stothers-Mason theorem is a counterpart of the $a b c$ conjecture in number theory, while its consequence described above is Fermat's last theorem for polynomials (see, e.g., [24,25]).

Fermat type functional equations, such as (1.1) and its generalizations, have been studied over many function fields [10,17,21] (see also, e.g., [15] and the references therein). For instance, if

$$
f_{1}^{n}+f_{2}^{n}+\cdots+f_{m}^{n}=1
$$

has a solution consisting of $m$ polynomials $f_{1}, f_{2}, \ldots, f_{m}$, then $n \leq m^{2}-m-1$. For rational, entire and meromorphic solutions the corresponding bounds are $n \leq m^{2}-2, n \leq m^{2}-m$ and $n \leq m^{2}-1$, respectively [17]. Hayman [18] calls the problem of finding the smallest $m=G_{0}(n)$ for which a solution of (1.2) exists as the Super-Fermat problem. A difference analogue of (1.2) was studied by the third author [26], who obtained similar bounds for a difference counterpart of (1.2) under certain conditions on the value distribution of solutions.

The purpose of this paper is to introduce a difference counterpart of the radical, and to use it to prove a difference analogue of the Stothers-Mason theorem, as well as a truncated version of the difference second main theorem for holomorphic curves. As applications we prove results on the non-existence of polynomial or non-polynomial entire solutions to difference Super-Fermat functional equations.

\section{Difference radical}

Let $p \not \equiv 0$ be a polynomial in $\mathbb{C}[z]$, and let $\kappa \in \mathbb{C} \backslash\{0\}$. We define the $\kappa$-difference radical $\operatorname{rãd}_{\kappa}(p)$ of $p$ as

$$
\operatorname{rãd}_{\kappa}(p(z))=\prod_{w \in \mathbb{C}}(z-w)^{d_{\kappa}(w)},
$$

where

$$
d_{\kappa}(w)=\operatorname{ord}_{w}(p)-\min \left\{\operatorname{ord}_{w}(p), \operatorname{ord}_{w+\kappa}(p)\right\}
$$

with $\operatorname{ord}_{w}(p) \geq 0$ being the order of zero of the polynomial $p$ at $w \in \mathbb{C}$. This corresponds to the way to define the usual radical $\operatorname{rad} p$ as

$$
\operatorname{rad} p(z)=\prod_{w \in \mathbb{C}}(z-w)^{d(w)},
$$


where, denoting by $p^{\prime}$ the derivative of $p$,

$$
d(w)=\operatorname{ord}_{w}(p)-\min \left\{\operatorname{ord}_{w}(p), \operatorname{ord}_{w}\left(p^{\prime}\right)\right\} \in\{0,1\} .
$$

Now, by defining $\tilde{n}_{\kappa}(p)=\operatorname{deg} \operatorname{rãd}_{\kappa}(p)$, it follows that $\tilde{n}_{\kappa}(p)$ is the number of zeros of $p$ appearing non-periodically with respect to the constant $\kappa$, where the multiplicities of the zeros are taken into account. In other words,

$$
\tilde{n}_{\kappa}(p)=\sum_{w \in \mathbb{C}}\left(\operatorname{ord}_{w}(p)-\min \left\{\operatorname{ord}_{w}(p), \operatorname{ord}_{w+\kappa}(p)\right\}\right) .
$$

For example, if $p$ has zeros of order 2,1 and 3 at $z_{0}, z_{0}+1$ and $z_{0}+2$, respectively, and no zero at $z_{0}+3$, then the zero of $p$ at $z_{0}$ is counted once in $\tilde{n}_{1}(p)$ and the zero at $z_{0}+2$ three times in $\tilde{n}_{1}(p)$, while the zero at $z_{0}+1$ is not counted in $\tilde{n}_{1}(p)$.

In addition, we define $\Delta_{\kappa} p=p(z+\kappa)-p(z)$, and use the notation $\operatorname{gcd}(p, q)$ to denote the greatest common divisor of $p$ and $q$ over $\mathbb{C}[z]$.

Lemma 2.1 Let $p \not \equiv 0$ be a polynomial in $\mathbb{C}[z]$. Then,

$$
p=\operatorname{gcd}\left(p, \Delta_{\kappa} p\right) \cdot \operatorname{rãd}_{\kappa}(p)
$$

and therefore

$$
\operatorname{deg} p=\operatorname{deg} \operatorname{gcd}\left(p, \Delta_{\kappa} p\right)+\tilde{n}_{\kappa}(p) .
$$

Proof We may write $p$ in the form

$$
p(z)=\gamma \prod_{i=1}^{m} \prod_{j=0}^{l_{i}}\left(z-\beta_{i}+j \kappa\right),
$$

where $\gamma \in \mathbb{C}$ and $l_{i} \in \mathbb{N} \cup\{0\}$. Note that the roots of $p$ are repeated in (2.2) the number of times according to their multiplicity, so the case $\beta_{i}=\beta_{k}, i \neq k$, is allowed. More precisely, for a zero of $p(z)$, if $\operatorname{ord}_{\beta}(p)>\operatorname{ord}_{\beta+\kappa}(p)$, then $\beta$ is entered $\operatorname{ord}_{\beta}(p)-\operatorname{ord}_{\beta+\kappa}(p)$ times as one of the ' $\left\{\beta_{i}\right\}$ ' in (2.2). If $\operatorname{ord}_{\beta}(p) \leq \operatorname{ord}_{\beta+\kappa}(p)$, then $\beta$ is not entered as one of the ' $\left\{\beta_{i}\right\}$ ' in (2.2). Moreover, we may assume in (2.2) that $\beta_{s} \neq \beta_{t}-\left(l_{t}+1\right) \kappa$ for any $s, t=1,2, \ldots, m$, since otherwise, we can combine two products as

$$
\prod_{j=0}^{l_{t}}\left(z-\beta_{t}+j \kappa\right) \cdot \prod_{j=0}^{l_{s}}\left(z-\beta_{s}+j \kappa\right)=\prod_{j=0}^{l_{t}+l_{s}+1}\left(z-\beta_{t}+j \kappa\right) .
$$

Now, by (2.2), the difference radical satisfies the simple representation

$$
\operatorname{rãd}_{\kappa}(p(z))=\prod_{i=1}^{m}\left(z-\beta_{i}\right) .
$$

In fact, for each $i \in\{1, \ldots, m\}$ we have

$$
d_{\kappa}\left(\beta_{i}-j \kappa\right)=\left\{\begin{array}{l}
1-\min \{1,1\}=0\left(1 \leq j \leq \ell_{i}\right), \\
1-\min \{1,0\}=1(j=0) .
\end{array}\right.
$$

From (2.2), we have

$$
p(z+\kappa)=\gamma \prod_{i=1}^{m} \prod_{j=0}^{l_{i}}\left(z-\beta_{i}+(j+1) \kappa\right),
$$


and so

$$
p(z)=\operatorname{gcd}(p(z), p(z+\kappa)) \cdot \operatorname{rãd}_{\kappa}(p(z)) .
$$

Since

$$
\operatorname{gcd}(p(z), p(z+\kappa))=\operatorname{gcd}(p(z), p(z+\kappa)-p(z))=\operatorname{gcd}\left(p(z), \Delta_{\kappa} p(z)\right),
$$

it follows that

$$
p(z)=\operatorname{gcd}\left(p(z), \Delta_{\kappa} p(z)\right) \cdot \operatorname{rãd}_{\kappa}(p(z)) .
$$

Therefore,

$$
\operatorname{deg} p=\operatorname{deg} \operatorname{gcd}\left(p, \Delta_{\kappa} p\right)+\operatorname{deg} \operatorname{rãd}_{\kappa}(p)=\operatorname{deg} \operatorname{gcd}\left(p, \Delta_{\kappa} p\right)+\tilde{n}_{\kappa}(p) .
$$

Remark By Lemma 2.1, we have

$$
\operatorname{rãd}_{\kappa}(p(z))=\frac{p(z)}{\operatorname{gcd}(p(z), p(z+\kappa))},
$$

which corresponds to the well known expression for the classical radical,

$$
\operatorname{rad} p(z)=\frac{p(z)}{\operatorname{gcd}\left(p(z), p^{\prime}(z)\right)} .
$$

In what follows we denote $\bar{n}(p)=\operatorname{deg}$ rad $p$ for the number of all the distinct roots of $p(z)$. Then, as Laeng [24] observed, we obtain the following properties for $\bar{n}$ :

(1) $\bar{n}(p) \leq \operatorname{deg} p$ for any $p(z) \in \mathbb{C}[z]$;

(2) $\bar{n}\left(p^{m}\right)=\bar{n}(p)$ for any $p(z) \in \mathbb{C}[z]$ and $m \in \mathbb{N}$;

(3) $\bar{n}(p q) \leq \bar{n}(p)+\bar{n}(q)$ for any $p(z), q(z) \in \mathbb{C}[z]$, where the equality holds exactly when $p(z)$ and $q(z)$ are relatively prime.

Of the $\kappa$-difference analogue $\tilde{n}_{\kappa}$, those properties change slightly but significantly. In fact, our definition (2.1) of $\tilde{n}_{\kappa}(p)$ implies:

(i) $\tilde{n}_{\kappa}(p) \leq \operatorname{deg} p$ for any $p(z) \in \mathbb{C}[z]$;

(2) $\tilde{n}_{\kappa}\left(p^{m}\right)=m \cdot \tilde{n}_{\kappa}(p)$ for any $p(z) \in \mathbb{C}[z]$ and $m \in \mathbb{N}$;

( $\tilde{3}) \tilde{n}_{\kappa}(p q) \leq \tilde{n}_{\kappa}(p)+\tilde{n}_{\kappa}(q)$ for any $p(z), q(z) \in \mathbb{C}[z]$, where the equality holds exactly when both rãd ${ }_{-\kappa}(p(z+\kappa))$ and $\operatorname{rãd}_{\kappa}(q(z))$, as well as rãd ${ }_{\kappa}(p(z))$ and rãd ${ }_{-\kappa}(q(z+\kappa))$ are relatively prime.

\section{Difference analogue of the Stothers-Mason theorem}

The Stothers-Mason theorem has been generalized in many different directions, for instance, to sums in one-dimensional function fields by Mason [28], by Voloch [43] and by Brownawell and Masser [2], to sums of pairwise relatively prime polynomials of several variables by Shapiro and Sparer [32], to sums in higher-dimensional function fields by Hsia and Wang [19], and to quantum deformations of polynomials by Vaserstein [41]. Motivated by the analogy between Diophantine approximation and Nevanlinna theory [42], the $a b c$ theorem has also been proven for complex entire functions by Van Frankenhuysen [39,40], for $p$-adic entire functions by $\mathrm{Hu}$ and Yang [20], and for non-Archimedean entire functions of several 
variables by Cherry and Toropu [5]. The following theorem is a difference analogue of the Stothers-Mason theorem, or in other words, a difference $a b c$ theorem for polynomials.

Theorem 3.1 Let $a, b$ and $c$ be relatively prime polynomials in $\mathbb{C}[z]$ such that

$$
a+b=c
$$

and such that $a, b$ and $c$ are not all constant. Then,

$$
\max \{\operatorname{deg} a, \operatorname{deg} b, \operatorname{deg} c\} \leq \tilde{n}_{\kappa}(a)+\tilde{n}_{\kappa}(b)+\tilde{n}_{\kappa}(c)-1,
$$

where $\kappa \in \mathbb{C} \backslash\{0\}$.

Proof Without loss of generality we may assume that $\max \{\operatorname{deg} a, \operatorname{deg} b, \operatorname{deg} c\}=\operatorname{deg} c$. From (3.1) it follows that

$$
\Delta_{\kappa} a+\Delta_{\kappa} b=\Delta_{\kappa} c
$$

Thus

$$
a \Delta_{\kappa} a+a \Delta_{\kappa} b=a \Delta_{\kappa} c
$$

and

$$
a \Delta_{\kappa} a+b \Delta_{\kappa} a=c \Delta_{\kappa} a .
$$

By subtracting (3.4) from (3.3), we have

$$
a \Delta_{\kappa} b-b \Delta_{\kappa} a=a \Delta_{\kappa} c-c \Delta_{\kappa} a,
$$

and so $\operatorname{gcd}\left(a, \Delta_{\kappa} a\right), \operatorname{gcd}\left(b, \Delta_{\kappa} b\right)$ and $\operatorname{gcd}\left(c, \Delta_{\kappa} c\right)$ are all factors of $a \Delta_{\kappa} b-b \Delta_{\kappa} a$. Since $a$, $b$ and $c$ are relatively prime, it follows that also $\operatorname{gcd}\left(a, \Delta_{\kappa} a\right), \operatorname{gcd}\left(b, \Delta_{\kappa} b\right)$ and $\operatorname{gcd}\left(c, \Delta_{\kappa} c\right)$ are relatively prime. Therefore,

$$
\operatorname{gcd}\left(a, \Delta_{\kappa} a\right) \operatorname{gcd}\left(b, \Delta_{\kappa} b\right) \operatorname{gcd}\left(c, \Delta_{\kappa} c\right)
$$

is a factor of $a \Delta_{\kappa} b-b \Delta_{\kappa} a$, which implies that

$$
\operatorname{deg} \operatorname{gcd}\left(a, \Delta_{\kappa} a\right)+\operatorname{deg} \operatorname{gcd}\left(b, \Delta_{\kappa} b\right)+\operatorname{deg} \operatorname{gcd}\left(c, \Delta_{\kappa} c\right) \leq \operatorname{deg} a+\operatorname{deg} b-1
$$

provided that $a \Delta_{\kappa} b-b \Delta_{\kappa} a \neq 0$. But if

$$
a \Delta_{\kappa} b-b \Delta_{\kappa} a=0,
$$

then $a \Delta_{\kappa} b=b \Delta_{\kappa} a$, and so $a$ is a factor of $b \Delta_{\kappa} a$. Since $a$ and $b$ have no common factors, it follows that $a$ is a factor of $\Delta_{\kappa} a$. This is only possible if $\Delta_{\kappa} a \equiv 0$. Similarly, under the assumption (3.6) it follows that $\Delta_{\kappa} b \equiv 0$ and $\Delta_{\kappa} c \equiv 0$, which contradicts the assumption of the theorem. Hence, (3.6) cannot hold and (3.5) is valid. By adding deg $c$ to both sides of (3.5) and reorganizing the terms, we have

$$
\begin{gathered}
\operatorname{deg} c \leq \operatorname{deg} a-\operatorname{deg} \operatorname{gcd}\left(a, \Delta_{\kappa} a\right)+\operatorname{deg} b-\operatorname{deg} \operatorname{gcd}\left(b, \Delta_{\kappa} b\right) \\
+\operatorname{deg} c-\operatorname{deg} \operatorname{gcd}\left(c, \Delta_{\kappa} c\right)-1 .
\end{gathered}
$$

The assertion follows by Lemma 2.1 . 
Example 3.2 We can see that the assertion of Theorem 3.1 is sharp by the example $a(z)=$ $(z+\alpha)(z+\alpha+\kappa), b(z)=-(z+\beta)(z+\beta+\kappa)$, and $c(z)=2(\alpha-\beta)(z+(\alpha+\beta+\kappa) / 2)$, where $\alpha, \beta \in \mathbb{C}$ such that $\beta \neq \alpha \neq \beta \pm \kappa$. Namely, then $a, b$ and $c$ are relatively prime polynomials in $\mathbb{C}[z]$ such that $a+b=c$, and such that none of the differences $\Delta_{\kappa} a, \Delta_{\kappa} b$ and $\Delta_{\kappa} c$ is identically zero. In addition, $\max \{\operatorname{deg} a, \operatorname{deg} b, \operatorname{deg} c\}=2, \widetilde{n}_{\kappa}(a)=1, \widetilde{n}_{\kappa}(b)=1$, $\tilde{n}_{\kappa}(c)=1$ and $\tilde{n}_{\kappa}(a)+\tilde{n}_{\kappa}(b)+\tilde{n}_{\kappa}(c)-1=2$.

Comparing the statements of the Stothers-Mason theorem and Theorem 3.1 raises the question of whether a stronger statement

$$
\max \{\operatorname{deg} a, \operatorname{deg} b, \operatorname{deg} c\} \leq \tilde{n}_{\kappa}(a b c)-1
$$

would hold in Theorem 3.1. The following coprime polynomial identity is extremal to the Stothers-Mason theorem but not to Theorem 3.1. Nevertheless, this proves the conclusion of Theorem 3.1 cannot be (3.7).

Example 3.3 Many polynomial identities are known to show the sharpness of the StothersMason theorem. Here we choose $16 z+(z+1)^{3}(z-3)=(z+3)(z-1)^{3}$ of them. Then rãd $-2\left(16 z(z+1)^{3}(z-3)(z+3)(z-1)^{3}\right)=z(z+1)^{2}(z+3)$ is of degree 4 and rãd $-4(16 z)=$ $z$, rãd-4 $\left((z+1)^{3}(z-3)\right)=(z+1)^{3}, \operatorname{rãd}_{-4}\left((z+3)(z-1)^{3}\right)=(z-1)^{2}(z+3)$. Of course, when we choose the step size $\kappa$ as -2 , each of the latter three radicals has the same degree as its input. On the other hand, one observes that other known identities such as $16 z^{3}+(z+1)(z-3)^{3}=(z+3)^{3}(z-1)$ or $8 z\left(z^{2}+1\right)+(z-1)^{4}=(z+1)^{4}$ do not work like the one that we chose here.

Example 3.2 shows that Theorem 3.1 is sharp when $\max \{\operatorname{deg} a, \operatorname{deg} b, \operatorname{deg} c\}=2$. In addition, choosing $\beta=\alpha+2 \kappa$ in Example 3.2 yields

$$
\tilde{n}_{\kappa}(a b c)-1=1<2=\max \{\operatorname{deg} a, \operatorname{deg} b, \operatorname{deg} c\},
$$

which is another counterexample showing that (3.2) cannot be replaced by (3.7) in the statement of Theorem 3.1. The following example demonstrates the sharpness of Theorem 3.1 for the case $\kappa=1$ and $\max \{\operatorname{deg} a, \operatorname{deg} b, \operatorname{deg} c\}=4$.

Example 3.4 Set $v=\frac{1+\sqrt{3} i}{2}$ noting that $v^{3}=-1$, and set $\alpha=1-v, \beta=v$. Define

$$
\begin{aligned}
& a(z)=A(z+\alpha)^{2}(z+\alpha+1)^{2}, \quad b(z)=-A(z+\beta)^{2}(z+\beta+1)^{2}, \\
& c(z)=z(z+1)(z+2) \quad \text { with } A=\frac{i}{4 \sqrt{3}} .
\end{aligned}
$$

Then $a, b$ and $c$ satisfy (3.1), and $\max \{\operatorname{deg} a, \operatorname{deg} b, \operatorname{deg} c\}=4, \tilde{n}_{1}(a)=2, \tilde{n}_{1}(b)=2$, $\tilde{n}_{1}(c)=1$.

The so-called Davenport inequality [7] for polynomials $f(z), g(z) \in \mathbb{C}[z]$ with $f(z)^{3} \not \equiv$ $g(z)^{2}$ (in answer to a question of Birch et al. [1]) is

$$
\operatorname{deg}\left(f(z)^{3}-g(z)^{2}\right) \geq \max \left\{\frac{1}{2} \operatorname{deg}(f(z)), \frac{1}{3} \operatorname{deg}(g(z))\right\}+1,
$$

to which Stothers [37] and independently Zannier [45] gave the extremal functions for each degree. The simplest example is

$$
\left\{\begin{array}{l}
f(z)=z^{2}+2 \\
g(z)=z^{3}+3 z
\end{array}\right.
$$


with $f(z)^{3}-g(z)^{2}=3 z^{2}+8$. For example, instead of (2, 3)-pair due to Stothers, Shioda [33] proposed to call a triple $\{f, g, h\}$ of polynomials a Davenport-Stothers triple (or a DS-triple) of order $m$ if it satisfies the following condition:

$$
f^{3}-g^{2}=h, \quad \operatorname{deg}(f)=2 m, \operatorname{deg}(g)=3 m, \operatorname{deg}(h)=m+1 .
$$

Then he continues: In this terminology, Stothers has proven among others the existence of $D S$-triples of order $m$ for every $m \geq 1$ and the finiteness of the number of essentially distinct $D S$-triples of order $m$. In fact, Davenport's elegant proof uses only linear algebra, while Stothers has generalized the Davenport inequality to the Stothers-Mason theorem along a geometric way to be mentioned shortly below. It should be mentioned here that Zannier gave Acknowledgement of priority [46] concerning his paper [45] that he recently discovered the paper [37] by Stothers, which actually covers part of his results, using a method of the same nature. This is almost a déjà vu for us and perhaps we should call this result the DavenportStothers-Zannier theorem in this note.

By applying Theorem 3.1 we give

$$
\operatorname{deg}(f(z-1) f(z) f(z+1)-g(z-1) g(z)) \geq \max \left\{\frac{1}{2} \operatorname{deg}(f(z)), \frac{1}{3} \operatorname{deg}(g(z))\right\}+1
$$

as a difference analogue of the Davenport-Stothers-Zannier inequality for $f(z), g(z) \in \mathbb{C}[z]$ with $f(z-1) f(z) f(z+1) \not \equiv g(z-1) g(z)$, to which

$$
\left\{\begin{array}{l}
f(z)=z(z+1)+2, \\
g(z)=z(z+1)(z+2)+3(z+1)
\end{array}\right.
$$

is an extremal example with $f(z-1) f(z) f(z+1)-g(z-1) g(z)=2(3 z(z+1)+8)$. At this moment we cannot however find any other difference analogues to DS-triples, unfortunately. This is because the method by Stothers and others is in a geometric way based on the Riemann-Hurwitz relation which gives crucial information for the case of equality as Shioda mentioned in [34]. In fact, as well as in the case of equality for the Stothers-Mason theorem, they consider a Belyi map of the Riemann sphere $\mathbb{P}(\mathbb{C})$ which is rational and unramified outside $\{0,1, \infty\}$. Such a strong method is not known yet in our difference setting, while we could find the above example through an ansatz by analogy to the simplest DS-triple as above.

In order to generalize Theorem 3.1 for $m+1$ polynomials we recall the definition of the Casorati determinant, and introduce the corresponding difference radical.

Let $a_{j}(z)(1 \leq j \leq m)$ be linearly independent functions over the field of periodic functions of period $\kappa \in \mathbb{C} \backslash\{0\}$. Then the Casoratian $\mathcal{C}_{\kappa}(z)$ of $a_{1}(z), \ldots, a_{m}(z)$, satisfying

$$
\mathcal{C}_{\kappa}(z)=\left|\begin{array}{cccc}
a_{1}(z) & a_{2}(z) & \cdots & a_{m}(z) \\
\Delta_{\kappa} a_{1}(z) & \Delta_{\kappa} a_{2}(z) & \cdots & \Delta_{\kappa} a_{m}(z) \\
& \ldots \ldots \cdots \cdots & \\
\cdots & \ldots & \\
\Delta_{\kappa}^{m-1} a_{1}(z) & \Delta_{\kappa}^{m-1} a_{2}(z) & \cdots & \Delta_{\kappa}^{m-1} a_{m}(z)
\end{array}\right|,
$$

does not vanish identically. Many of the properties of Casoratians are similar to those of Wronskians, in part due to the correspondence of

$$
\Delta_{\kappa}(z(z+\kappa) \cdots(z+(n-1) \kappa))=n \kappa \frac{z(z+\kappa) \cdots(z+(n-1) \kappa)}{z}
$$


to $\frac{d}{d z}\left(z^{n}\right)=n \frac{z^{n}}{z}$, which implies the degree relation

$$
\operatorname{deg}\left(D^{j} p(z)\right)=\operatorname{deg} p(z)-j, \quad 0 \leq j \leq \operatorname{deg} p(z)
$$

for any $p(z) \in \mathbb{C}[z]$ with both operators $D=d / d z$ and $\Delta_{\kappa}$.

The difference radical of truncation level $q$ for $p(z) \in \mathbb{C}[z]$, denoted by $\operatorname{rãd}_{\kappa}^{[q]}(p)$, is defined by

$$
\begin{aligned}
\operatorname{rãd}_{\kappa}^{[q]}(p(z)) & =\frac{p(z)}{\operatorname{gcd}\left\{p(z), \Delta_{\kappa} p(z), \Delta_{\kappa}^{2} p(z), \ldots, \Delta_{\kappa}^{q} p(z)\right\}} \\
& =\frac{p(z)}{\operatorname{gcd}\{p(z), p(z+\kappa), p(z+2 \kappa), \ldots, p(z+q \kappa)\}} .
\end{aligned}
$$

Then we define

$$
\begin{aligned}
\tilde{n}_{\kappa}^{[q]}(p) & =\operatorname{deg} \operatorname{rãd}_{\kappa}^{[q]}(p) \\
& =\sum_{w \in \mathbb{C}}\left(\operatorname{ord}_{w}(p)-\min _{0 \leq j \leq q}\left\{\operatorname{ord}_{w+j \kappa}(p)\right\}\right) .
\end{aligned}
$$

Note that when $q=1$ these definitions reduce to the ones given in Sect. 2, that is, we have $\operatorname{rãd}_{\kappa}^{[1]}(p(z))=\operatorname{rãd}_{\kappa}(p(z))$ and $\tilde{n}_{\kappa}^{[1]}(p)=\tilde{n}_{\kappa}(p)$. Writing $p(z)$ in the form (2.2) for $l_{i} \in \mathbb{N} \cup\{0\}(i=1, \ldots, m)$, we have

$$
\begin{aligned}
\tilde{n}_{\kappa}^{[q]}(p) & =\sum_{i=1}^{m}\left(l_{i}+1\right)-\sum_{i=1}^{m}\left|L_{i} \cap\left(L_{i}+1\right) \cap \cdots \cap\left(L_{i}+q\right)\right| \\
& =\sum_{i=1}^{m}\left(l_{i}+1\right)-\sum_{i=1}^{m} \max \left\{0, l_{i}-q+1\right\} \\
& =\sum_{i=1}^{m} \min \left\{l_{i}+1, q\right\},
\end{aligned}
$$

where $\left|L_{i} \cap\left(L_{i}+1\right) \cap \cdots \cap\left(L_{i}+q\right)\right|$ denotes the number of elements in the intersection of the set $L_{i}=\left\{0,1, \ldots, l_{i}\right\}$ and its translations $L_{i}+k:=\left\{k, 1+k, \ldots, l_{i}+k\right\}$ for $k=1,2, \ldots, q$.

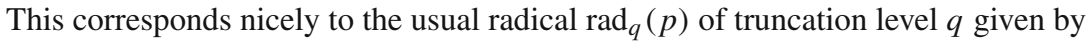

$$
\operatorname{rad}_{q}(p(z))=\frac{p(z)}{\operatorname{gcd}\left\{p(z), p^{\prime}(z), p^{\prime \prime}(z), \ldots, p^{(q)}(z)\right\}} .
$$

In fact, Hu and Yang [21] use the notation $r_{q}(p)$ for the degree of $\operatorname{rad}_{q}(p)$ of truncation level $q$ given by

$$
r_{q}\left(c \prod_{j=1}^{l}\left(z-\alpha_{j}\right)^{m_{j}}\right)=\sum_{j=1}^{l} \min \left\{m_{j}, q\right\},
$$

where $\alpha_{1}, \ldots, \alpha_{l}$ are distinct, $m_{j} \in \mathbb{N}(j=1, \ldots, l)$ and $c \neq 0$. By the above expression of $\operatorname{rad}_{q}(p(z))$ for $p(z)=c \prod_{j=1}^{l}\left(z-\alpha_{j}\right)^{m_{j}}$, one obtains indeed

$$
\begin{aligned}
\operatorname{deg} \operatorname{rad}_{q}(p) & =\operatorname{deg} p-\operatorname{deg} \operatorname{gcd}\left(p, p^{\prime}, \ldots, p^{(q)}\right) \\
& =\sum_{j=1}^{l} m_{j}-\sum_{j=1}^{l} \max \left\{0, m_{j}-q\right\}=\sum_{j=1}^{l} \min \left\{m_{j}, q\right\}=r_{q}(p) .
\end{aligned}
$$


The following theorem extends Theorem 3.1 for $m+1$ polynomials, see, for example, $[8,21,32]$ for the differential analogue. In the statement of the theorem we have chosen to follow the form used by Shapiro and Sparer [32], instead of the one due to de Bondt [8] with weaker assumptions. Extending the theorem using de Bondt's approach is beyond the scope of our paper, and it is left as an open question.

Theorem 3.5 Let $a_{1}, \ldots, a_{m+1}$ be pairwise relatively prime polynomials in $\mathbb{C}[z]$ such that

$$
a_{1}+\cdots+a_{m}=a_{m+1},
$$

and such that $a_{1}, \ldots, a_{m}$ are linearly independent over $\mathbb{C}$. Then,

$$
\max _{1 \leq i \leq m+1}\left\{\operatorname{deg} a_{i}\right\} \leq \sum_{i=1}^{m+1} \tilde{n}_{\kappa}^{[m-1]}\left(a_{i}\right)-\frac{1}{2} m(m-1),
$$

where we denote

$$
\tilde{n}_{\kappa}^{[m-1]}\left(a_{i}\right)=\operatorname{deg} \operatorname{rãd}_{\kappa}^{[m-1]}\left(a_{i}\right)=\sum_{w \in \mathbb{C}}\left(\operatorname{ord}_{w}\left(a_{i}\right)-\min _{0 \leq j \leq m-1}\left\{\operatorname{ord}_{w+j \kappa}\left(a_{i}\right)\right\}\right)
$$

and $\kappa \in \mathbb{C} \backslash\{0\}$.

Proof Now we consider the Casoratian $\mathcal{C}_{\kappa}(z) \not \equiv 0$ of $a_{1}(z), \ldots, a_{m}(z)$. Let $z_{0}$ be a zero of some $a_{i}(z)$ with $1 \leq i \leq m+1$. Then $\mathcal{C}_{\kappa}(z)$ has also a zero at $z=z_{0}$ of multiplicity not smaller than

$$
\min _{0 \leq j \leq m-1}\left\{\operatorname{ord}_{z 0}\left(a_{i}(z+j \kappa)\right)\right\}
$$

Therefore, under the assumption of the pairwise relative primeness we see that

$$
q(z):=\prod_{i=1}^{m+1} \operatorname{gcd}\left(a_{i}(z), a_{i}(z+\kappa), \ldots, a_{i}(z+(m-1) \kappa)\right)
$$

divides $\mathcal{C}_{K}(z)$, so that there exists a polynomial $p(z) \in \mathbb{C}[z]$ satisfying $\mathcal{C}_{K}(z)=p(z) q(z)$. Note that the degree of $q(z)$ is not less than

$$
\sum_{i=1}^{m+1} \sum_{w \in \mathbb{C}} \min _{0 \leq j \leq m-1}\left\{\operatorname{ord}_{w}\left(a_{i}(z+j \kappa)\right)\right\}=\sum_{i=1}^{m+1}\left[\sum_{w \in \mathbb{C}} \operatorname{ord}_{w}\left(a_{i}\right)-\tilde{n}_{\kappa}^{[m-1]}\left(a_{i}\right)\right]
$$

by means of the notation (3.10).

On the other hand, the degree of $\mathcal{C}_{\kappa}(z)$ is never beyond any sum of distinct $m$ of the $\operatorname{deg} a_{i}(z)(1 \leq i \leq m+1)$ minus $\sum_{\ell=0}^{m-1} \ell=m(m-1) / 2$ as the sum of $\operatorname{deg}\left(\Delta_{\kappa}^{\ell} a_{i_{\nu}}\right)$ for the mutually distinct $m$ integers $i_{v} \in\{1, \ldots, m, m+1\}$. Hence we obtain

$$
\begin{aligned}
\min _{1 \leq k \leq m+1} \sum_{1 \leq i \leq m+1, i \neq k} \operatorname{deg} a_{i}-\frac{1}{2} m(m-1) & \geq \sum_{i=1}^{m+1}\left[\sum_{w \in \mathbb{C}} \operatorname{ord}_{w}\left(a_{i}\right)-\tilde{n}_{\kappa}^{[m-1]}\left(a_{i}\right)\right] \\
& =\sum_{i=1}^{m+1} \operatorname{deg} a_{i}-\sum_{i=1}^{m+1} \tilde{n}_{\kappa}^{[m-1]}\left(a_{i}\right) .
\end{aligned}
$$


This implies our desired estimate

$$
\max _{1 \leq i \leq m+1}\left\{\operatorname{deg} a_{i}\right\} \leq \sum_{i=1}^{m+1} \tilde{n}_{\kappa}^{[m-1]}\left(a_{i}\right)-\frac{1}{2} m(m-1) .
$$

Note that $\tilde{n}_{\kappa}^{[m-1]}\left(a_{i}\right)$ in the above estimate cannot be replaced by $\tilde{n}_{\kappa}\left(a_{i}\right)=\operatorname{deg} \operatorname{rand}_{\kappa}\left(a_{i}\right)$ to obtain

$$
\max _{1 \leq i \leq m+1}\left\{\operatorname{deg} a_{i}\right\} \leq \sum_{i=1}^{m+1} \operatorname{deg} \operatorname{rãd}_{\kappa}\left(a_{i}\right)-\frac{1}{2} m(m-1) .
$$

By definition

$$
\tilde{n}_{\kappa}^{[m-1]}\left(a_{i}\right)-\tilde{n}_{\kappa}\left(a_{i}\right)=\sum_{w \in \mathbb{C}}\left[\min \left\{\operatorname{ord}_{w}\left(a_{i}\right), \operatorname{ord}_{w+\kappa}\left(a_{i}\right)\right\}-\min _{0 \leq j \leq m-1}\left\{\operatorname{ord}_{w+j \kappa}\left(a_{i}\right)\right\}\right]
$$

is always non-negative so that $\tilde{n}_{\kappa}^{[m-1]}\left(a_{i}\right) \geq \tilde{n}_{\kappa}\left(a_{i}\right)$. Example 3.6 below shows that (3.11) does not hold in general when $m>2$. If one wished to use the radicals rãd ${ }_{\kappa}\left(a_{i}\right)$ in (3.9), it is possible to use such estimates as

$$
\begin{aligned}
\tilde{n}_{\kappa}^{[m-1]}\left(a_{i}\right)= & \sum_{w \in \mathbb{C}}\left\{\operatorname{ord}_{w}\left(a_{i}\right)-\min \left\{\operatorname{ord}_{w}\left(a_{i}\right), \operatorname{ord}_{w+\kappa}\left(a_{i}\right), \ldots, \operatorname{ord}_{w+(m-1) \kappa}\left(a_{i}\right)\right\}\right\} \\
= & \sum_{w \in \mathbb{C}} \max \left\{0, \operatorname{ord}_{w}\left(a_{i}\right)-\operatorname{ord}_{w+\kappa}\left(a_{i}\right), \ldots, \operatorname{ord}_{w}\left(a_{i}\right)-\operatorname{ord}_{w+(m-1) \kappa}\left(a_{i}\right)\right\} \\
\leq & \sum_{w \in \mathbb{C}} \max \left\{0, \operatorname{ord}_{w}\left(a_{i}\right)-\operatorname{ord}_{w+\kappa}\left(a_{i}\right)\right\}+\cdots \\
& \quad+\sum_{w \in \mathbb{C}} \max \left\{0, \operatorname{ord}_{w}\left(a_{i}\right)-\operatorname{ord}_{w+(m-1) \kappa}\left(a_{i}\right)\right\} \\
= & \sum_{w \in \mathbb{C}}\left\{\operatorname{ord}_{w}\left(a_{i}\right)-\min \left\{\operatorname{ord}_{w}\left(a_{i}\right), \operatorname{ord}_{w+\kappa}\left(a_{i}\right)\right\}\right\}+\cdots \\
& +\sum_{w \in \mathbb{C}}\left\{\operatorname{ord}_{w}\left(a_{i}\right)-\min \left\{\operatorname{ord}_{w}\left(a_{i}\right), \operatorname{ord}_{w+(m-1) \kappa}\left(a_{i}\right)\right\}\right\} \\
= & \tilde{n}_{\kappa}\left(a_{i}\right)+\cdots+\tilde{n}_{(m-1) \kappa}\left(a_{i}\right)=\sum_{j=1}^{m-1} \tilde{n}_{j \kappa}\left(a_{i}\right),
\end{aligned}
$$

which can be sharp when $m=2$ but the following example shows this is a crude estimate for our purposes.

Example 3.6 Given $c \in \mathbb{C} \backslash\{0\}$, we have the identity

$$
\left(z^{2}-c^{2}\right)\left\{(z+1)^{2}-c^{2}\right\}+\left(z^{2}+c^{2}\right)\left\{(z+1)^{2}+c^{2}\right\}=2 z^{2}(z+1)^{2}+2 c^{4} .
$$

Thus we have a solution $\left(a_{1}, a_{2}, a_{3}, a_{4}\right)$ to the equation

$$
a_{1}+a_{2}+a_{3}=a_{4}
$$

with $m=3$, such that $\left\{a_{1}, a_{2}, a_{3}\right\}$ is a linear independent system of pairwise relatively prime polynomials by requiring $c \neq 0, \pm 1, \pm \sqrt{-1}$. In fact, we put

$$
p_{1}(z)=z^{2}-c^{2}, \quad p_{2}(z)=z^{2}+c^{2}, \quad p_{3}(z)=\sqrt{-2} z^{2}
$$


and

$$
a_{1}(z)=p_{1}(z) p_{1}(z+1), \quad a_{2}(z)=p_{2}(z) p_{2}(z+1), \quad a_{3}(z)=p_{3}(z) p_{3}(z+1),
$$

and $a_{4}(z)=2 c^{4}$. Then we have $\max _{1 \leq i \leq 4}\left\{\operatorname{deg} a_{i}\right\}=4$ and

$$
\sum_{i=1}^{3} \operatorname{deg} \operatorname{rãd}_{1}\left(a_{i}\right)=2+2+2=6
$$

by observing the zeros of $a_{i}(i=1,2,3)$, respectively. Hence it is not possible for us to replace $\sum_{i=1}^{m+1} \tilde{n}_{\kappa}^{[m-1]}\left(a_{i}\right)$ in the above estimate by $\sum_{i=1}^{m+1} \tilde{n}_{\kappa}\left(a_{i}\right)$, since $\frac{1}{2} m(m-1)=3$. On the other hand, this example gives

$$
\sum_{i=1}^{4} \tilde{n}_{1}^{[2]}\left(a_{i}\right)=4+4+4=12,
$$

so that this is far from an example to confirm whether our estimate is sharp, unfortunately. For this purpose, one needs to consider such an example that the $a_{i}(z)$ are of the form $a_{i}(z)=p_{i}(z) p_{i}(z+\kappa) \cdots p_{i}(z+(n-1) \kappa)$ for $n \geq m$ so that $\min _{0 \leq j \leq m-1}\left\{\operatorname{ord}_{w+j \kappa}\left(a_{i}\right)\right\}$ is positive at a zero of $a_{i}(z)$. Note that this quantity is always zero when $n<m$ and the zeros of $p_{i}(z)$ appear non-periodically with respect to $\kappa$.

The following example observes the acuity of Theorem 3.5 in the case when $m=3$ with $\kappa=1$.

Example 3.7 Define

$$
\begin{aligned}
& a_{1}(z)=A(z+\alpha)(z+\alpha+1)(z+\alpha+2)^{2}, \\
& a_{2}(z)=B(z+\beta)(z+\beta+1)(z+\beta+2)^{2}, \\
& a_{3}(z)=-(A+B) z(z+1)(z+2)(z+3) .
\end{aligned}
$$

By simple computations, we see that $a_{1}(z)+a_{2}(z)+a_{3}(z)$ reduces to a polynomial, say $a_{4}(z)$, of degree at most 1 when $\alpha \neq-1 / 4$ and

$$
\beta=\frac{2 \alpha+1}{8 \alpha-2}, \quad B=-\frac{(4 \alpha-1)^{2}}{3} A .
$$

Indeed, we have with (3.12)

$$
\begin{aligned}
a_{1}(z)+a_{2}(z)+a_{3}(z)= & \frac{A\left(8 \alpha^{2}-4 \alpha-1\right)\left(32 \alpha^{3}-8 \alpha^{2}+4 \alpha-1\right)}{4(1-4 \alpha)^{2}} z \\
& +\frac{A\left(8 \alpha^{2}-4 \alpha-1\right)\left(32 \alpha^{4}+160 \alpha^{3}-8 \alpha^{2}+8 \alpha-3\right)}{16(1-4 \alpha)^{2}} .
\end{aligned}
$$

We can choose $\alpha$ so that $a_{j}(z), j=1,2,3,4$ are pairwise relatively prime. Then $a_{j}$ satisfy (3.8), and $\max _{1 \leq j \leq 4}\left\{\operatorname{deg} a_{j}\right\}=4, \tilde{n}_{1}^{[2]}\left(a_{1}\right)=3, \tilde{n}_{1}^{[2]}\left(a_{2}\right)=3, \tilde{n}_{1}^{[2]}\left(a_{3}\right)=2$. If $a_{4}(z)$ is nonconstant, then $\tilde{n}_{1}^{[2]}\left(a_{4}\right)=1$ which gives $4 \leq 6$, which is not enough to show the sharpness of Theorem 3.5 for the case $m=3$. Next, we set $\alpha=\frac{i}{2 \sqrt{2}}$ and $A=2-\sqrt{2} i$. Then $\beta=-\frac{i}{2 \sqrt{2}}, B=2+\sqrt{2} i$, and $a_{4}(z)$ reduces to a constant $-\frac{9}{16}$, which gives a somewhat sharper estimate $4 \leq 5$ for Theorem 3.5 when $a_{m+1}$ is a constant. 


\section{Polynomial solutions of difference Super-Fermat functional equations}

A factorial polynomial is defined as

$$
t^{\bar{n}}=t(t+1) \cdots(t+n-1) .
$$

We extend this notation for the factorial of a polynomial $p$ in $\mathbb{C}[z]$ as

$$
[p]_{\kappa}^{\bar{n}}=p(z) p(z+\kappa) \cdots p(z+(n-1) \kappa),
$$

where the shift $\kappa \in \mathbb{C} \backslash\{0\}$.

As a consequence of Theorem 3.1 we obtain the following result on the non-existence of polynomial solutions to a difference Fermat functional equation.

Theorem 4.1 Let $\kappa \in \mathbb{C} \backslash\{0\}, n \in \mathbb{N}$ and $a, b, c \in \mathbb{C}[z]$, not all constant. If $[a]_{\kappa}^{\bar{n}},[b]_{\kappa}^{\bar{n}}$ and $[c]_{\kappa}^{\bar{n}}$ are relatively prime and satisfy

$$
[a]_{\kappa}^{\bar{n}}+[b]_{\kappa}^{\bar{n}}=[c]_{\kappa}^{\bar{n}},
$$

then $n \leq 2$. If at least one of $a, b$ and $c$ is constant, then $n=1$.

Proof Suppose first that none of $a, b$ and $c$ is constant. If (4.1) holds, then by Theorem 3.1, we have

$$
\begin{aligned}
\operatorname{deg}[a]_{\kappa}^{\bar{n}} & \leq \max \left\{\operatorname{deg}[a]_{\kappa}^{\bar{n}}, \operatorname{deg}[b]_{\kappa}^{\bar{n}}, \operatorname{deg}[c]_{\kappa}^{\bar{n}}\right\} \\
& \leq \tilde{n}_{\kappa}\left([a]_{\kappa}^{\bar{n}}\right)+\tilde{n}_{\kappa}\left([b]_{\kappa}^{\bar{n}}\right)+\tilde{n}_{\kappa}\left([c]_{\kappa}^{\bar{n}}\right)-1 \\
& \leq \operatorname{deg} a+\operatorname{deg} b+\operatorname{deg} c-1 .
\end{aligned}
$$

Since $\operatorname{deg}[a]_{\kappa}^{\bar{n}}=n \operatorname{deg} a$, it follows that

$$
n \operatorname{deg} a \leq \operatorname{deg} a+\operatorname{deg} b+\operatorname{deg} c-1 .
$$

By repeating the same argument for $b$ and $c$ instead of $a$, we have

$$
n \operatorname{deg} b \leq \operatorname{deg} a+\operatorname{deg} b+\operatorname{deg} c-1
$$

and

$$
n \operatorname{deg} c \leq \operatorname{deg} a+\operatorname{deg} b+\operatorname{deg} c-1 .
$$

By combining (4.2), (4.3) and (4.4), it follows that

$$
n(\operatorname{deg} a+\operatorname{deg} b+\operatorname{deg} c) \leq 3(\operatorname{deg} a+\operatorname{deg} b+\operatorname{deg} c)-3,
$$

and so $n \leq 2$.

Assume now that at least one of $a, b$ and $c$ is constant. Then by (4.1) exactly one of them, say $c$, is constant. Then, by (4.2) and (4.3), we have

$$
n(\operatorname{deg} a+\operatorname{deg} b) \leq 2(\operatorname{deg} a+\operatorname{deg} b)-2,
$$

which implies that $n \leq 1$.

The following example shows that the assertion of Theorem 4.1 is sharp. 
Example 4.2 Choosing $\kappa=1$ and defining

$$
\begin{aligned}
& a(z)=z^{2} \\
& b(z)=-\frac{i}{2}\left(\sqrt{2} z^{2}+2 z-\sqrt{2}\right), \\
& c(z)=-\frac{1}{2}\left(\sqrt{2} z^{2}-2 z-\sqrt{2}\right),
\end{aligned}
$$

it follows that $[a]_{1}^{\overline{2}},[b]_{1}^{\overline{2}}$ and $[c]_{1}^{\overline{2}}$ have no common factors, and they satisfy (4.1) with $n=2$.

Remark It is natural that the assertions of Lemma 2.1 and of Theorems 3.1 and 4.1 remain valid in a more general setting where the polynomials are in $k[z]$, where $k$ is any algebraically closed field of characteristic zero. For the case when the characteristic $p$ of $k$ is positive, we have

$$
\Delta_{p / 2}(z(z+p / 2))=(z+p / 2)(z+p)-z(z+p / 2)=p(z+p / 2) \equiv 0
$$

but $z(z+p / 2)$ is not identically a constant. Such a complex structure with $p>0$ encourages us to restrict our coefficient field to be of characteristic zero, even if the above singularity can be avoided by a choice $\kappa \neq p / 2$ of the shift of $\Delta_{\kappa}$.

The following theorem extends Theorem 4.1 to equations with arbitrarily many terms.

Theorem 4.3 If $m \geq 2$ and $p_{1}, \ldots, p_{m+1}$ are non-constant polynomials in $\mathbb{C}[z]$ such that $\left[p_{1}\right]_{\kappa}^{\bar{n}},\left[p_{2}\right]_{\kappa}^{\bar{n}}, \ldots,\left[\bar{p}_{m+1}\right]_{\kappa}^{\bar{n}}$ are pairwise relatively prime and satisfy

$$
\left[p_{1}\right]_{\kappa}^{\bar{n}}+\left[p_{2}\right]_{\kappa}^{\bar{n}}+\cdots+\left[p_{m}\right]_{\kappa}^{\bar{n}}=\left[p_{m+1}\right]_{\kappa}^{\bar{n}}
$$

for some $\kappa \in \mathbb{C} \backslash\{0\}$ and $n \in \mathbb{N}$, then

$$
n \leq m^{2}-1-\frac{m(m-1)}{2 \max _{1 \leq i \leq m+1}\left\{\operatorname{deg} p_{i}\right\}} .
$$

Proof We may assume, without loss of generality, that $\left[p_{1}\right]_{\kappa}^{\bar{n}},\left[p_{2}\right]_{\kappa}^{\bar{n}}, \ldots,\left[p_{m}\right]_{\kappa}^{\bar{n}}$ are linearly independent. For otherwise we may eliminate some of the polynomials from (4.5) to obtain a shorter equation, which is of the same form, but contains only linearly independent terms. Assume that $m>n$. Since for all $m \geq 2$ the right hand side of (4.6) is always at least $m$, the inequality itself follows.

Now we suppose that $n \geq m$. By using Theorem 3.5 we obtain

$$
n \cdot \max _{1 \leq i \leq m+1} \operatorname{deg} p_{i} \leq \sum_{i=1}^{m+1} \operatorname{deg} \operatorname{rãd}_{\kappa}^{[m-1]}\left(\left[p_{i}\right]_{\kappa}^{\bar{n}}\right)-\frac{1}{2} m(m-1) .
$$


Further we have

$$
\begin{aligned}
\operatorname{deg} \operatorname{rãd}_{\kappa}^{[m-1]}\left(\left[p_{i}\right]_{\kappa}^{\bar{n}}\right) & \\
= & \sum_{w \in \mathbb{C}}\left\{\operatorname{ord}_{w}\left(\left[p_{i}\right]_{\kappa}^{\bar{n}}\right)\right. \\
& \left.\quad-\min \left\{\operatorname{ord}_{w}\left(\left[p_{i}\right]_{\kappa}^{\bar{n}}\right), \operatorname{ord}_{w+\kappa}\left(\left[p_{i}\right]_{\kappa}^{\bar{n}}\right), \ldots, \operatorname{ord}_{w+(m-1) \kappa}\left(\left[p_{i}\right]_{\kappa}^{\bar{n}}\right)\right\}\right\} \\
= & \sum_{w \in \mathbb{C}}\left\{\operatorname{ord}_{w}\left(p_{i}\right)+\operatorname{ord}_{w+\kappa}\left(p_{i}\right)+\cdots+\operatorname{ord}_{w+(n-1) \kappa}\left(p_{i}\right)\right. \\
& -\min \left\{\operatorname{ord}_{w}\left(p_{i}\right)+\operatorname{ord}_{w+\kappa}\left(p_{i}\right)+\cdots+\operatorname{ord}_{w+(n-1) \kappa}\left(p_{i}\right),\right. \\
& \operatorname{ord}_{w+\kappa}\left(p_{i}\right)+\operatorname{ord}_{w+2 \kappa}\left(p_{i}\right)+\cdots+\operatorname{ord}_{w+n \kappa}\left(p_{i}\right), \ldots, \\
& \left.\left.\operatorname{ord}_{w+(m-1) \kappa}\left(p_{i}\right)+\operatorname{ord}_{w+m \kappa}\left(p_{i}\right)+\cdots+\operatorname{ord}_{w+(n+m-2) \kappa}\left(p_{i}\right)\right\}\right\} \\
\leq & \sum_{w \in \mathbb{C}}\left\{\operatorname{ord}_{w}\left(p_{i}\right)+\operatorname{ord}_{w+\kappa}\left(p_{i}\right)+\cdots+\operatorname{ord}_{w+(m-2) \kappa}\left(p_{i}\right)\right\} \\
\leq & (m-1) \operatorname{deg} p_{i},
\end{aligned}
$$

since, when $m \leq n$, it follows

$$
\begin{gathered}
\min \left\{\operatorname{ord}_{w}\left(p_{i}\right)+\operatorname{ord}_{w+\kappa}\left(p_{i}\right)+\cdots+\operatorname{ord}_{w+(n-1) \kappa}\left(p_{i}\right),\right. \\
\quad \operatorname{ord}_{w+\kappa}\left(p_{i}\right)+\operatorname{ord}_{w+2 \kappa}\left(p_{i}\right)+\cdots+\operatorname{ord}_{w+n \kappa}\left(p_{i}\right), \ldots, \\
\left.\operatorname{ord}_{w+(m-1) \kappa}\left(p_{i}\right)+\operatorname{ord}_{w+m \kappa}\left(p_{i}\right)+\cdots+\operatorname{ord}_{w+(n+m-2) \kappa}\left(p_{i}\right)\right\} \\
\geq \operatorname{ord}_{w+(m-1) \kappa}\left(p_{i}\right)+\operatorname{ord}_{w+m \kappa}\left(p_{i}\right)+\cdots+\operatorname{ord}_{w+(n-1) \kappa}\left(p_{i}\right) .
\end{gathered}
$$

Therefore (4.7) gives

$$
n \cdot \max _{1 \leq i \leq m+1} \operatorname{deg} p_{i} \leq(m+1)(m-1) \max _{1 \leq i \leq m+1} \operatorname{deg} p_{i}-\frac{1}{2} m(m-1),
$$

which implies the assertion in the case $n \geq m$.

Example 4.4 We consider the sharpness of the inequality (4.6) in the case $m=2$. Let us first look at the case where the maximal degree of the polynomial solutions of (4.5) is one. In this case it can be seen by a direct substitution of arbitrary linear polynomials into (4.5) that such solutions are never relatively prime when $n=2$. If the maximal degree of the polynomial solutions is two, then by Theorem 4.3 we have $n \leq 5 / 2$. In Example 4.2 we have given a solution for the Eq. (4.5) with $m=2$ and $n=2$, which is optimal in this case, since $n$ is an integer.

Theorem 4.3 immediately implies an upper bound for $n$ in (4.5), which only depends on $m$ as follows.

Corollary 4.5 If the assumptions of Theorem 4.3 are satisfied, then $n \leq m^{2}-2$.

Choosing $m=2$ in Corollary 4.5 implies the first assertion of Theorem 4.1, namely that $n \leq 2$.

The final two results of this section deal with another canonical form of difference Fermat functional equations.

Theorem 4.6 If $m \geq 2$ and $p_{1}, \ldots, p_{m}$ are non-constant polynomials in $\mathbb{C}[z]$ such that $\left[p_{1}\right]_{\kappa}^{\bar{n}},\left[p_{2}\right]_{\kappa}^{\bar{n}}, \ldots,\left[p_{m}\right]_{\kappa}^{\bar{n}}$ are pairwise relatively prime and satisfy

$$
\left[p_{1}\right]_{\kappa}^{\bar{n}}+\left[p_{2}\right]_{\kappa}^{\bar{n}}+\cdots+\left[p_{m}\right]_{\kappa}^{\bar{n}}=1
$$


for some $\kappa \in \mathbb{C} \backslash\{0\}$ and $n \in \mathbb{N}$, then

$$
n \leq m^{2}-m-\frac{m(m-1)}{2 \max _{1 \leq i \leq m}\left\{\operatorname{deg} p_{i}\right\}} .
$$

Proof From Theorem 3.5 we have

$$
n \cdot \max _{1 \leq i \leq m} \operatorname{deg} p_{i} \leq \sum_{i=1}^{m} \operatorname{deg} \operatorname{rãd}_{\kappa}^{[m-1]}\left(\left[p_{i}\right]_{\kappa}^{\bar{n}}\right)-\frac{1}{2} m(m-1),
$$

and so a similar discussion as in the proof of Theorem 4.3 implies the assertion.

Corollary 4.7 If the assumptions of Theorem 4.6 are satisfied, then $n \leq m^{2}-m-1$.

If at least one of the polynomials in the Eq. (4.5) is constant, then (4.5) reduces into (4.8). In particular, when $m=2$, Corollary 4.7 then implies the second assertion of Theorem 4.1, namely that $n=1$.

\section{Non-polynomial entire solutions of difference Super-Fermat functional equations}

In this section we extend the results obtained in Sect. 4 for the case of entire solutions of hyper-order strictly less than one. The hyper-order of an entire function $g$ is defined as

$$
\rho_{2}(g)=\limsup _{r \rightarrow \infty} \frac{\log ^{+} \log ^{+} T(r, g)}{\log r},
$$

where $T(r, g)$ is the Nevanlinna characteristic function of $g$. For $\kappa \in \mathbb{C} \backslash\{0\}$ we denote by $\mathcal{P}_{\kappa}^{1}$ the field of period $\kappa$ meromorphic functions of hyper-order strictly less than one.

In the case of hyper-order $\geq 1$, for an arbitrary integer $n \geq 2$ there exists a transcendental entire function $f(z)$ such that $[f]_{\kappa}^{\bar{n}}$ reduces to a constant. For example, consider $f(z)=$ $\exp \left(\pi(z) \omega^{z / \kappa}\right)$ where $\pi(z)$ is a $\kappa$-periodic entire function of order $\rho(\geq 1)$ and $\omega \neq 1$ is an $n$th root of unity. Then we have $\rho_{2}(f)=\rho$ and

$$
[f]_{\kappa}^{\bar{n}}=\prod_{j=0}^{n-1} f(z+j \kappa)=\prod_{j=0}^{n-1} \exp \left(\pi(z) \omega^{z / \kappa} \omega^{j}\right)=\exp \left(\pi(z) \omega^{z / \kappa} \sum_{j=0}^{n-1} \omega^{j}\right) \equiv e^{0}=1 .
$$

Here we have applied an existence theorem of prime periodic entire functions by Ozawa [30, Theorems 1 and 2], where he proved that for arbitrarily given $\kappa \neq 0$ and $\rho(1 \leq \rho \leq \infty)$, there exists a $\kappa$-periodic entire function $\pi(z)$ of order $\rho$. Examples of the type (5.1) are in stark contrast to the behavior of polynomials, and so we want to rule them out in this note.

Proposition 5.1 Let $m \geq 2$ and let $f_{1}, \ldots, f_{m}$ be non-constant entire functions such that $\rho_{2}\left(f_{i}\right)<1$ for all $i \in\{1, \ldots, m\}$, and such that $\left[f_{1}\right]_{\kappa}^{\bar{n}},\left[f_{2}\right]_{\kappa}^{\bar{n}}, \ldots,\left[f_{m}\right]_{\underline{\kappa}}^{\bar{n}}$ are linearly independent over $\mathcal{P}_{\kappa}^{1}$ and furthermore $\left[f_{1}\right]_{\kappa}^{\bar{n}},\left[f_{2}\right]_{\kappa}^{\bar{n}}, \ldots,\left[f_{m}\right]_{\kappa}^{\bar{n}}$ and $\left[f_{m+1}\right]_{\kappa}^{\bar{n}}$ have no common zeros. If

$$
\left[f_{1}\right]_{\kappa}^{\bar{n}}+\left[f_{2}\right]_{\kappa}^{\bar{n}}+\cdots+\left[f_{m}\right]_{\kappa}^{\bar{n}}=\left[f_{m+1}\right]_{\kappa}^{\bar{n}}
$$

for some $\kappa \in \mathbb{C} \backslash\{0\}$ and $n \in \mathbb{N}$, then $n \leq m^{2}-1$. 
In Proposition 5.1 we have assumed that the entire functions $\left[f_{1}\right]_{\kappa}^{\bar{n}},\left[f_{2}\right]_{\kappa}^{\bar{n}}, \ldots,\left[f_{m+1}\right]_{\kappa}^{\bar{n}}$ do not have common zeros in an analogy of the assumption of pairwise relative primeness of the polynomials in Theorem 4.3.

Proposition 5.2 Let $m \geq 2$ and let $f_{1}, \ldots, f_{m}$ be non-constant entire functions such that $\rho_{2}\left(f_{i}\right)<1$ for all $i \in\{1, \ldots, m\}$, and such that $\left[f_{1}\right]_{\kappa}^{\bar{n}},\left[f_{2}\right]_{\kappa}^{\bar{n}}, \ldots,\left[f_{m}\right]_{\kappa}^{\bar{n}}$ are linearly independent over $\mathcal{P}_{\kappa}^{1}$. If

$$
\left[f_{1}\right]_{\kappa}^{\bar{n}}+\left[f_{2}\right]_{\kappa}^{\bar{n}}+\cdots+\left[f_{m}\right]_{\kappa}^{\bar{n}}=1,
$$

for some $\kappa \in \mathbb{C} \backslash\{0\}$ and $n \in \mathbb{N}$, then $n \leq m^{2}-m$.

For the differential counterparts of Propositions 5.1 and 5.2, see, for instance [15,17].

Example 5.3 It is well known that the Fermat functional equation

$$
f(z)^{2}+g(z)^{2}=1
$$

has a trigonometric solution

$$
\left\{\begin{array}{l}
f(z)=\cos z=\frac{1}{2}\left(e^{i z}+e^{-i z}\right), \\
g(z)=\sin z=\frac{1}{2 i}\left(e^{i z}-e^{-i z}\right) .
\end{array}\right.
$$

Corresponding to Proposition 5.2, the difference Fermat functional equation

$$
f(z) f(z+1)+g(z) g(z+1)=1
$$

has a trigonometric solution

$$
\left\{\begin{array}{l}
f(z)=\sqrt[4]{2} i \sin \frac{\pi}{4} z=\frac{1}{\sqrt[4]{8}}\left\{\left(\frac{1+i}{\sqrt{2}}\right)^{z}+\left(\frac{1+i}{\sqrt{2}}\right)^{-z}\right\}, \\
g(z)=\sqrt[4]{2} \cos \frac{\pi}{4} z=\frac{1}{\sqrt[4]{8}}\left\{\left(\frac{1+i}{\sqrt{2}}\right)^{z}-\left(\frac{1+i}{\sqrt{2}}\right)^{-z}\right\} .
\end{array}\right.
$$

The Fermat functional equation

$$
f(z)^{3}+g(z)^{3}=1
$$

has an elliptic solution

$$
\left\{\begin{array}{l}
f(z)=\frac{\sqrt{3}+\wp^{\prime}(z)}{2 \sqrt{3} \wp(z)} \\
g(z)=\frac{\sqrt{3}-\wp^{\prime}(z)}{2 \sqrt{3} \wp(z)},
\end{array}\right.
$$

where $\wp$ is the Weierstrass $\wp$-function satisfying $\left(\wp^{\prime}\right)^{2}=4 \wp^{3}-1$. This parametrization of the Fermat curve is given by Gross [14], while the Weierstrass map $\left(\sqrt[3]{4} \wp, \wp^{\prime}\right)$ parametrizes the elliptic curve $y^{2}=x^{3}-1$. These uniformizations are equivalent, while functions appearing in both expressions behave differently from the value-distribution point of view. In fact, the four elliptic functions $\left(\frac{\sqrt{3} \pm \wp^{\prime}(z)}{2 \sqrt{3} \wp(z)}\right)^{3}, 4 \wp^{3}$ and $-\left(\wp^{\prime}\right)^{2}$ are all unramified outside $\{0, \infty, 1\}$. The former two functions attain the three values with the same multiplicity 3 , while those multiplicities of the latter two function are 2, 6 and 3, both cases of which are extremal to Nevanlinna's inequality. Geometrically, we distinguish Gross' and Weiestrass' expressions in the above by the way of triangular tilings for the Euclidean plane. 
Corresponding to Proposition 5.1, an elementary computation shows that the difference Fermat functional equation

$$
f(z-1) f(z) f(z+1)+g(z-1) g(z) g(z+1)=1
$$

has an elliptic solution

$$
\left\{\begin{array}{l}
f(z)=\frac{1}{\wp\left(h\left(z-z_{0}\right)\right)}+1 \\
g(z)=\frac{1}{\wp\left(h\left(z-z_{0}\right)\right)}-1
\end{array}\right.
$$

where $\wp$ is the Weierstrass $\wp$-function satisfying $\left(\wp^{\prime}\right)^{2}=4 \wp^{3}-2$ and $z_{0}$ and $h$ are constants with $\wp(h)=0$.

Before we can prove Propositions 5.1 and 5.2, we need to introduce tools to handle entire functions. In particular, we will consider an extension of the notion of difference radical for entire functions, and define the corresponding Nevanlinna counting functions.

The order of a holomorphic curve $g: \mathbb{C} \rightarrow \mathbb{P}^{n}$ is defined by

$$
\sigma(g)=\limsup _{r \rightarrow \infty} \frac{\log ^{+} T_{g}(r)}{\log r},
$$

where $\log ^{+} x=\max \{0, \log x\}$ for all $x \geq 0$, and

$$
T_{g}(r):=\int_{0}^{2 \pi} u\left(r e^{i \theta}\right) \frac{d \theta}{2 \pi}-u(0), \quad u(z)=\sup _{k \in\{0, \ldots, n\}} \log \left|g_{k}(z)\right|,
$$

is the Cartan characteristic function of $g$ with the reduced representation $g=\left[g_{0}: \cdots: g_{n}\right]$. Similarly, the hyper-order of $g: \mathbb{C} \rightarrow \mathbb{P}^{n}$ is

$$
\varsigma(g)=\limsup _{r \rightarrow \infty} \frac{\log ^{+} \log ^{+} T_{g}(r)}{\log r} .
$$

The following lemma [16, Lemma 8.3] is a useful tool in dealing with shifts in characteristic and Nevanlinna counting functions.

Lemma 5.4 [16] Let $T:[0,+\infty) \rightarrow[0,+\infty)$ be a non-decreasing continuous function and let $s \in(0, \infty)$. If the hyper-order of $T$ is strictly less than one, i.e.,

$$
\limsup _{r \rightarrow \infty} \frac{\log \log T(r)}{\log r}=\varsigma<1
$$

and $\delta \in(0,1-\varsigma)$, then

$$
T(r+s)=T(r)+o\left(\frac{T(r)}{r^{\delta}}\right),
$$

where $r$ runs to infinity outside of a set of finite logarithmic measure.

We give a comparison estimate of $T(r, f(z+\kappa))$ in terms of $T(r \pm|\kappa|, f(z))$, as well as of $N(r, f(z+\kappa))$ in terms of $N(r \pm|\kappa|, f(z))$. In the proof of the following lemma we implement an alternate approach to the estimates discussed in [12, p. 47]. 
Lemma 5.5 Let $f \not \equiv 0$ be meromorphic in $\mathbb{C}$ and let $\kappa \in \mathbb{C} \backslash\{0\}$. Then

$$
(1-o(1)) T(r-|\kappa|, f(z)) \leq T(r, f(z+\kappa)) \leq(1+o(1)) T(r+|\kappa|, f(z))
$$

and

$$
(1-o(1)) N(r-|\kappa|, f(z)) \leq N(r, f(z+\kappa)) \leq(1+o(1)) N(r+|\kappa|, f(z))
$$

for all $r \geq|\kappa|$.

Proof We will first prove (5.3). Recall the usual definition of the counting function

$$
N(r, f)=\int_{0}^{r} \frac{n(t, f)-n(0, f)}{t} d t+n(0, f) \log r .
$$

Here $n(r, f)$ is the number of poles of $f$ according to order in the disc of radius $r$ centred at the origin, so that

$$
n(r-|\kappa|, f(z)) \leq n(r, f(z+\kappa)) \leq n(r+|\kappa|, f(z))
$$

for all $r \geq \kappa$. On the other hand, a version of l'Hospital's rule (see, for example, [38]) implies both

$$
\begin{aligned}
\limsup _{r \rightarrow \infty} \frac{N(r, f(z+\kappa))}{N(r+|\kappa|, f(z))} & \leq \limsup _{r \rightarrow \infty} \frac{\frac{d}{d \log r} N(r, f(z+\kappa))}{\frac{d}{d \log r} N(r+|\kappa|, f(z))} \\
& =\limsup _{r \rightarrow \infty} \frac{n(r, f(z+\kappa))}{\frac{r}{r+|\kappa|} n(r+|\kappa|, f(z))},
\end{aligned}
$$

and

$\liminf _{r \rightarrow \infty} \frac{N(r, f(z+\kappa))}{N(r-|\kappa|, f(z))} \geq \liminf _{r \rightarrow \infty} \frac{\frac{d}{d \log r} N(r, f(z+\kappa))}{\frac{d}{d \log r} N(r-|\kappa|, f(z))}=\liminf _{r \rightarrow \infty} \frac{n(r, f(z+\kappa))}{\frac{r}{r-|\kappa|} n(r-|\kappa|, f(z))}$,

which, together with (5.4), yield (5.3).

To prove (5.2), we recall the Cartan identity [3] (see also, e.g., [6, Theorem 1.13.1])

$$
T(r, f)=\int_{0}^{2 \pi} N\left(r, \frac{1}{f-e^{i \theta}}\right) \frac{d \theta}{2 \pi}+C,
$$

where $C \in \mathbb{R}$ is independent of $r$. Similarly as above, we have

$$
\begin{aligned}
\int_{0}^{2 \pi} n\left(r-|\kappa|, \frac{1}{f(z)-e^{i \theta}}\right) \frac{d \theta}{2 \pi} & \leq \int_{0}^{2 \pi} n\left(r, \frac{1}{f(z+\kappa)-e^{i \theta}}\right) \frac{d \theta}{2 \pi} \\
& \leq \int_{0}^{2 \pi} n\left(r+|\kappa|, \frac{1}{f(z)-e^{i \theta}}\right) \frac{d \theta}{2 \pi}
\end{aligned}
$$

for all $r \geq \kappa$, and

$$
\limsup _{r \rightarrow \infty} \frac{T(r, f(z+\kappa))}{T(r+|\kappa|, f(z))} \leq \limsup _{r \rightarrow \infty} \frac{\int_{0}^{2 \pi} n\left(r, \frac{1}{f(z+\kappa)-e^{i \theta}}\right) \frac{d \theta}{2 \pi}}{\frac{r}{r+|\kappa|} \int_{0}^{2 \pi} n\left(r+|\kappa|, \frac{1}{f(z)-e^{i \theta}}\right) \frac{d \theta}{2 \pi}},
$$

as well as

$$
\liminf _{r \rightarrow \infty} \frac{T(r, f(z+\kappa))}{T(r-|\kappa|, f(z))} \geq \liminf _{r \rightarrow \infty} \frac{\int_{0}^{2 \pi} n\left(r, \frac{1}{f(z+\kappa)-e^{i \theta}}\right) \frac{d \theta}{2 \pi}}{\frac{r}{r-|\kappa|} \int_{0}^{2 \pi} n\left(r-|\kappa|, \frac{1}{f(z)-e^{i \theta}}\right) \frac{d \theta}{2 \pi}},
$$


which imply (5.2).

Remark One may notice that the second part can be simplified a little by observing the Ahlfors-Shimizu characteristic function instead of applying Cartan's identity to Nevanlinna's $T(r, f)$. See [29, Sections 3 and 4 of Chapter VI] or [31, Section 6], for example, for some related discussions on those characteristic functions as well as on the quantity $\int_{0}^{2 \pi} n\left(r, \frac{1}{f-e^{i \theta}}\right) \frac{d \theta}{2 \pi}$.

We denote by $\bar{D}\left(z_{0}, s\right)=\left\{z \in \mathbb{C}:\left|z-z_{0}\right| \leq s\right\}$ the closed disc of radius $s>0$ centred at $z_{0} \in \mathbb{C}$. We define, as in [6], the order $\operatorname{ord}_{\zeta}(f)$ of a meromorphic function $f$ at $\zeta \in \mathbb{C}$ as the unique $\mu \in \mathbb{Z}$ such that

$$
\lim _{z \rightarrow \zeta} \frac{f(z)}{(z-\zeta)^{\mu}} \in \mathbb{C} \backslash\{0\} .
$$

With this notation $\operatorname{ord}_{\zeta}(f)>0$ if and only if $f$ has a zero of order $\operatorname{ord}_{\zeta}(f)$ at $\zeta$, and $\operatorname{ord}_{\zeta}(f)<0$ if and only if $f$ has a pole of order $-\operatorname{ord}_{\zeta}(f)$ at $\zeta$. We also adopt the notation $\operatorname{ord}_{\zeta}^{+}(f)=\max \left\{0, \operatorname{ord}_{\zeta}(f)\right\}$ and $\operatorname{ord}_{\zeta}^{-}(f)=\max \left\{0,-\operatorname{ord}_{\zeta}(f)\right\}$. Now, given $q \in \mathbb{N}$, we define

$$
\tilde{n}_{\kappa}^{[q]}\left(r, \frac{1}{f}\right)=\sum_{w \in \bar{D}(0, r)}\left(\operatorname{ord}_{w}^{+}(f)-\min _{0 \leq j \leq q}\left\{\operatorname{ord}_{w+j \kappa}^{+}(f)\right\}\right)
$$

as a difference analogue of the truncated counting function for the zeros of $f$. The corresponding integrated counting function is defined in the usual way as

$$
\tilde{N}_{\kappa}^{[q]}\left(r, \frac{1}{f}\right)=\int_{0}^{r} \frac{\tilde{n}_{\kappa}^{[q]}(t, 1 / f)-\tilde{n}_{\kappa}^{[q]}(0,1 / f)}{t} d t+\tilde{n}_{\kappa}^{[q]}(0,1 / f) \log r .
$$

Also, by defining

$$
\lambda_{2}(f)=\limsup _{r \rightarrow \infty} \frac{\log ^{+} \log ^{+} N\left(r, \frac{1}{f}\right)}{\log r},
$$

it follows that $\lambda_{2}(f) \leq \rho_{2}(f)$. The following lemma demonstrates how the truncation works with the counting function (5.5).

Lemma 5.6 Let $f \not \equiv 0$ be entire, let $\kappa \in \mathbb{C} \backslash\{0\}$ and let $n, q \in \mathbb{N}$. If $\lambda_{2}(f)<1$, then

$$
\tilde{N}_{\kappa}^{[q]}\left(r, \frac{1}{[f]_{\kappa}^{\bar{n}}}\right) \leq q N\left(r, \frac{1}{f}\right)+o(N(r, 1 / f))
$$

as $r \rightarrow \infty$ outside of an exceptional set of finite logarithmic measure.

Proof Suppose first that $n>q$. Then by definition

$$
\begin{aligned}
\widetilde{n}_{\kappa}^{[q]}\left(r, \frac{1}{[f]_{\kappa}^{\bar{n}}}\right) & =\sum_{w \in \bar{D}(0, r)}\left(\sum_{i=0}^{n-1} \operatorname{ord}_{w}^{+} f(z+i \kappa)-\min _{j \in\{0, \ldots, q\}}\left\{\sum_{i=0}^{n-1} \operatorname{ord}_{w+j \kappa}^{+} f(z+i \kappa)\right\}\right) \\
& =\sum_{w \in \bar{D}(0, r)}\left(\sum_{i=0}^{n-1} \operatorname{ord}_{w+i \kappa}^{+}(f)-\min _{j \in\{0, \ldots, q\}}\left\{\sum_{i=0}^{n-1} \operatorname{ord}_{w+(i+j) \kappa}^{+}(f)\right\}\right) .
\end{aligned}
$$


Each term in the minimum on the right hand side of equation (5.6) contains the sum $\sum_{i=q}^{n-1} \operatorname{ord}_{w+i \kappa}^{+}(f)$. To see this, we may write $\sum_{i=0}^{n-1} \operatorname{ord}_{w+(i+j) \kappa}^{+}(f)=\sum_{k=j}^{n+j-1} \operatorname{ord}_{w+k \kappa}^{+}(f)$, if necessary. Therefore, it follows by (5.6) that

$$
\begin{aligned}
\tilde{n}_{\kappa}^{[q]}\left(r, \frac{1}{[f]_{\kappa}^{\bar{n}}}\right) & \leq \sum_{w \in \bar{D}(0, r)}\left(\sum_{i=0}^{n-1} \operatorname{ord}_{w+i \kappa}^{+}(f)-\sum_{i=q}^{n-1} \operatorname{ord}_{w+i \kappa}^{+}(f)\right) \\
& =\sum_{w \in \bar{D}(0, r)}\left(\sum_{i=0}^{q-1} \operatorname{ord}_{w+i \kappa}^{+}(f)\right) \\
& =\sum_{i=0}^{q-1} n\left(r, \frac{1}{f(z+i \kappa)}\right) .
\end{aligned}
$$

By integrating (5.7) it follows that

$$
\tilde{N}_{\kappa}^{[q]}\left(r, \frac{1}{[f]_{\kappa}^{\bar{n}}}\right) \leq \sum_{i=0}^{q-1} N\left(r, \frac{1}{f(z+i \kappa)}\right),
$$

since $n>q$. If $n \leq q$, the inequality (5.8) holds trivially, so in fact we have (5.8) for all $n \in \mathbb{N}$. The assertion now follows by Lemmas 5.4 and 5.5.

The following result is a truncated version of the second main theorem for differences proved in [16], and it can be viewed as a counterpart of Theorem 3.5 for entire functions. For the second main theorem of Cartan, we refer to [4] as well as [15].

Theorem 5.7 Let $g_{1}, \ldots, g_{m}$ be $m \geq 2$ entire functions with no common zeros, linearly independent over $\mathcal{P}_{\kappa}^{1}$, and let $g_{m+1}=g_{1}+\cdots+g_{m}$. If the holomorphic curve $g=\left[g_{1}\right.$ : $\left.\cdots: g_{m}\right]$ satisfies $\varsigma(g)<1$, then

$$
T_{g}(r) \leq \sum_{j=1}^{m+1} \widetilde{N}_{\kappa}^{[m-1]}\left(r, \frac{1}{g_{j}}\right)+o\left(\frac{T_{g}(r)}{r^{1-\varsigma(g)-\varepsilon}}\right),
$$

where $\kappa \in \mathbb{C} \backslash\{0\}, \varepsilon>0$, and $r \rightarrow \infty$ outside of an exceptional set of finite logarithmic measure.

Proof Denote by $\mathcal{C}_{\kappa}\left(g_{1}, \ldots, g_{m}\right)$ the Casoratian of $g_{1}, \ldots, g_{m}$, and define

$$
G=g_{1} \cdots g_{m+1} / \mathcal{C}_{\kappa}\left(g_{1}, \ldots, g_{m}\right) .
$$

Suppose $w$ is a zero of $G$. We assert that

$$
\operatorname{ord}_{w}^{+}(G) \leq \sum_{j=1}^{m+1}\left(\operatorname{ord}_{w}^{+}\left(g_{j}\right)-\min _{i \in\{0, \ldots, m-1\}}\left\{\operatorname{ord}_{w+i \kappa}^{+}\left(g_{j}\right)\right\}\right) .
$$

To confirm this, we write

$$
\frac{1}{G}=\frac{\epsilon}{g_{l_{0}}} \cdot\left|\begin{array}{cccc}
1 & 1 & \ldots & 1 \\
\frac{g_{l_{1}}(z+\kappa)}{g_{l_{1}}(z)} & \frac{g_{l_{2}}(z+\kappa)}{g_{l_{2}}(z)} & \ldots & \frac{g_{l_{m}}(z+\kappa)}{g_{l_{m}}(z)} \\
\vdots & \vdots & \ddots & \vdots \\
\frac{g_{l_{1}(z+(m-1) \kappa)}}{g_{l_{1}}(z)} & \frac{g_{l_{2}(z+(m-1) \kappa)}}{g_{l_{2}}(z)} & \ldots & \frac{g_{l_{m}}(z+(m-1) \kappa)}{g_{l_{m}}(z)}
\end{array}\right|,
$$


where $\epsilon \in\{ \pm 1\}$, and the indexes $\left\{l_{1}, \ldots, l_{m}\right\} \subset\{1, \ldots, m+1\}$ and $l_{0} \in\{1, \ldots, m+$ $1\} \backslash\left\{l_{1}, \ldots, l_{m}\right\}$ depend on $z$ so that $g_{l_{0}}(z) \neq 0$ for all $z \in \mathbb{C}$. We see that the zeros of $G$ are the poles of some of $g_{l_{j}}(z+i \kappa) / g_{l_{j}}(z), 1 \leq j \leq m, 1 \leq i \leq m-1$. The maximal order of pole among the $j$ th column in the determinant above is given by

$$
\max _{i \in\{0, \ldots, m-1\}}\left\{\operatorname{ord}_{w}^{+}\left(g_{l_{j}}\right)-\left\{\operatorname{ord}_{w+i \kappa}^{+}\left(g_{l_{j}}\right)\right\}\right\}=\operatorname{ord}_{w}^{+}\left(g_{l_{j}}\right)-\min _{i \in\{0, \ldots, m-1\}}\left\{\operatorname{ord}_{w+i \kappa}^{+}\left(g_{l_{j}}\right)\right\} .
$$

Since $g_{l_{0}}(z) \neq 0$ for all $z \in \mathbb{C}$, we obtain (5.10), and hence we have

$$
n\left(r, \frac{1}{G}\right) \leq \sum_{j=1}^{m+1} \tilde{n}_{\kappa}^{[m-1]}\left(r, \frac{1}{g_{j}}\right) .
$$

The assertion now follows by integrating (5.11) and applying [16, Theorem 2.1].

\section{Proof of Propositions 5.1 and 5.2}

By denoting

$$
N(r)=\sup _{j \in\{1, \ldots, m+1\}} N\left(r, \frac{1}{g_{j}}\right),
$$

where $g_{j}=\left[f_{j}\right]_{\kappa}^{\bar{n}}, j=1, \ldots, m+1$, and applying (5.9) we have

$$
N(r) \leq \sum_{j=1}^{m+1} \tilde{N}_{\kappa}^{[m-1]}\left(r, \frac{1}{\left[f_{j}\right]_{\kappa}^{\bar{n}}}\right)+o\left(T_{g}(r)\right)
$$

as $r \rightarrow \infty$ outside of an exceptional set $E$ of finite logarithmic measure. By defining

$$
M(r)=\sup _{j \in\{1, \ldots, m+1\}} N\left(r, \frac{1}{f_{j}}\right),
$$

we have, by applying Lemma 5.5 to the functions $f_{j}(z+i \kappa), i=0, \ldots, n-1, j=$ $1, \ldots, m+1$,

$$
n(1-o(1)) M(r-n|\kappa|) \leq N(r) \leq n(1+o(1)) M(r+n|\kappa|)
$$

for all $r \geq n|\kappa|$, and so by Lemma 5.4 it follows that

$$
N(r)=n M(r)+o(M(r))
$$

as $r \rightarrow \infty$ outside of an exceptional set $F$ of finite logarithmic measure. Therefore, Lemma 5.6 yields

$$
\begin{aligned}
n M(r) & =N(r)+o(M(r)) \\
& \leq \sum_{j=1}^{m+1}(m-1) N\left(r, \frac{1}{f_{j}}\right)+o(M(r)) \\
& \leq(m+1)(m-1) M(r)+o(M(r))
\end{aligned}
$$

as $r \rightarrow \infty$ outside of $E \cup F$, and so $n \leq m^{2}-1$. Thus Proposition 5.1 is proved. The assertion of Proposition 5.2, $n \leq m(m-1)$, follows by applying (5.9), but now with $g_{j}=\left[f_{j}\right]_{\kappa}^{\bar{n}}$, $j=1, \ldots, m$, and $g_{m+1}=1$. 


\section{Discussion}

The Oesterlé-Masser $a b c$ conjecture for integers states that given $\varepsilon>0$, there exists a number $K(\varepsilon)$ having the following property: For any nonzero relatively prime integers $a, b, c$ such that $a+b=c$, we have

$$
\max \{|a|,|b|,|c|\} \leq K(\varepsilon)(\operatorname{rad}(a b c))^{1+\varepsilon} .
$$

It should be noticed that Stewart and Tijdeman [36] proved that the conjecture would be false without the $\varepsilon$ and with an arbitrary fixed $K(\varepsilon)>1$. For example, for $K(\varepsilon)=1$ we have

$$
\max \{|a|,|b|,|c|\}>\operatorname{rad}(a b c)
$$

with $a=1, b=2^{m+2}\left(2^{m}-1\right)$ and $c=\left(2^{m+1}-1\right)^{2}$ for $m \in \mathbb{N}$. Further, in his survey article [25], Lang introduced to us an intelligible example due to Wojtek Jastrzebowski and Dan Spielman showing that the ratio $\max \{|a|,|b|,|c|\} / \operatorname{rad}(a b c)$ can be arbitrarily large, that is, $1+\left(3^{2^{n}}-1\right)=3^{2^{n}}$ since $2^{n}$ divides $3^{2^{n}}-1$ for any $n \in \mathbb{N}$. He remarks that a similar example is available by using a prime $p$ instead of 2 and an integer $\equiv 1 \bmod p$ instead of 3 , respectively.

On the other hand, the statement

$$
\max \{|a|,|b|,|c|\} \leq(\operatorname{rad}(a b c))^{2}
$$

seems to be widely believed true. In each of the above cases, the radical of $a b c$ is powered uniformly on the prime factors $p_{j}$, that is, independently of the exponents of $d_{j}(a b c)$ in the factorization of

$$
a b c= \pm \prod_{p_{j} \mid a b c} p_{j}^{d_{j}(a b c)}
$$

even in the case when $d_{j}(a b c)=1$ for some $j$. Thus, one might expect to replace $(\operatorname{rad}(a b c))^{2}$ by the 2 -truncated radical

$$
\operatorname{rad}^{[2]}(a b c)=\prod_{p_{j} \mid a b c} p_{j}^{d_{j}^{[2]}(a b c)}
$$

with $d_{j}^{[2]}(a b c)=\min \left\{d_{j}(a b c), 2\right\}$, for example. Unfortunately, as for usual radical, there are exceptional $a b c$ triples such that

$$
\max \{|a|,|b|,|c|\}>\operatorname{rad}^{[2]}(a b c)
$$

for this radical, too. In fact, we can obtain this inequality with some of the known exceptional $a b c$ triples such as

$$
\begin{gathered}
\left(2,3^{10} \cdot 109,23^{5}\right),\left(1,2 \cdot 3^{7}, 5^{4} \cdot 7\right),\left(7^{3}, 3^{10}, 2^{11} \cdot 29\right) \text { and } \\
\left(5^{3}, 2^{9} \cdot 3^{17} \cdot 13^{2}, 11^{5} \cdot 17 \cdot 31^{3} \cdot 137\right),
\end{gathered}
$$

which are listed with their 'ontdekker', Eric Reyssat, Benne de Weger and te Riele, Montgomery, for example, in Geuze and de Smit [11, Tabel 1] as well as at de Smit's website [9]. Note that the Jastrzebowski-Spielman example works here again, while the triple $\left(1,2^{m+2}\left(2^{m}-1\right),\left(2^{m+1}-1\right)^{2}\right)$ gives the opposite inequality.

Another possible replacement of $\operatorname{rad}(a b c)$ may be a sort of its difference analogue that we have studied in this note for functions. In other words, one can now ask the following: 
similarly as the Stothers-Mason theorem corresponds to the $a b c$ conjecture, what would be the analogue of Theorem 3.1 in number theory?

Let $p_{1}=2, p_{2}=3, p_{3}=5, \ldots, p_{i}, \ldots$ denote the collection of all prime numbers listed in increasing order, and let $x \in \mathbb{N}$. Then we can write $x$ in the unique form

$$
x=\prod_{i=1}^{\infty} p_{i}^{\operatorname{ord}_{i}(x)}
$$

where $\operatorname{ord}_{i}(x) \in \mathbb{N} \cup\{0\}$ for all $i \in \mathbb{N}$. Now we define the difference radical for $x$ by

$$
\operatorname{rãd}(x)=\prod_{i=1}^{\infty} p_{i}^{d(i)},
$$

where

$$
d(i)=\operatorname{ord}_{i}(x)-\min \left\{\operatorname{ord}_{i}(x), \operatorname{ord}_{i+1}(x)\right\} .
$$

Recall that the sizes of $\operatorname{rãd}(x)$ and $\operatorname{rad}(x)$ are not in a fixed order such as $\operatorname{rãd}(2 \cdot 3)<\operatorname{rad}(2 \cdot 3)$ and $\operatorname{rãd}\left(2^{2}\right)>\operatorname{rad}\left(2^{2}\right)$. We have observed the same property for the corresponding radicals in the polynomial ring. Here let us observe one typical difference among their properties by seeing an interesting question on the ordinary radical in the integer ring that is known as the Erdös-Woods conjecture made by Woods in his Ph. D. Thesis [44]: There exists an absolute constant $k>2$ such that for every positive integers $x$ and $y$, the conditions

$$
\operatorname{rad}(x+i)=\operatorname{rad}(y+i), \quad i=0,1,2, \ldots, k-1
$$

imply $x=y$. Erdös gave a counterexample $x=75=3 \cdot 5^{2}$ and $y=1215=3^{5} \cdot 5$ showing the necessity of the assumption $k>2$, since $76=2^{2} \cdot 19$ and $1216=2^{6} \cdot 19$. By the idea used above, one also finds the pair $x=2^{m+1}-2$ and $y=2^{m+1}\left(2^{m+1}-2\right)$ for each $m \in \mathbb{N}$, since $\operatorname{rad}\left(2^{m+1}\left(2^{m+1}-2\right)+1\right)=\operatorname{rad}\left(2^{m+1}-1\right)$. On the other hand, any such examples seem not possible at all for our difference radical. For example, we have $\operatorname{rãd}\left(3 \cdot 5^{2}\right)=5^{2}, \operatorname{rãd}\left(3^{5} \cdot 5\right)=3^{4} \cdot 5, \operatorname{rãd}\left(2^{2} \cdot 19\right)=2^{2} \cdot 19$ and $\operatorname{rãd}\left(2^{6} \cdot 19\right)=2^{6} \cdot 19$, and also rãd $\left(2^{m+1}-2\right) \neq \operatorname{rãd}\left(2^{m+1}\left(2^{m+1}-2\right)\right), \operatorname{rãd}\left(2^{m+1}-1\right) \neq \operatorname{rãd}\left(\left(2^{m+1}-1\right)^{2}\right)$ for each $m \in \mathbb{N}$, respectively. Of course, it would be imprudent of us to claim that rãd has a higher accuracy than rad does only with this kind of simple comparison. It is reasonable for us to say instead that each of both radicals demonstrates their own ability in leveling the multiple factors of target in the ring under observation, that is, of polynomials or of integers, respectively. Hence we believe that the new radical rãd gives at least another indicator of the factorization of integers and thus a difference analogue of the $a b c$ conjecture, which might have been stated as follows: For every positive real number $\varepsilon$, there exist only finitely many triples $(a, b, c)$ of coprime positive integers, with $a+b=c$, such that

$$
c>(\operatorname{rãd}(a b c))^{1+\varepsilon} .
$$

But we remember a property of difference radicals in the polynomial ring, that is, $\operatorname{rãd}(a b c)$ needs to be replaced by $\operatorname{rãd}(a) \operatorname{rãd}(b) \operatorname{rãd}(c)$ in Theorem 3.1. This choice must bring a significant change here, since the ratios

$$
c / \operatorname{rãd}(a b c) \text { and } c /(\operatorname{rãd}(a) \operatorname{rãd}(b) \operatorname{rãd}(c))
$$


can sometimes have large differences. For example, take the exceptional $a b c$ triple $\left(283,5^{11}\right.$. $13^{2}, 2^{8} \cdot 3^{8} \cdot 17^{3}$ ) discovered by Browkin Brzezinki, Nitaj as listed in [11, Tabel 1] and estimate

$$
\frac{2^{8} \cdot 3^{8} \cdot 17^{3}}{\operatorname{rãd}\left(2^{8} \cdot 3^{8} \cdot 5^{11} \cdot 13^{2} \cdot 17^{3} \cdot 283\right)}=\frac{2^{8} \cdot 3^{8} \cdot 17^{3}}{2^{0} \cdot 3^{0} \cdot 5^{11} \cdot 13^{0} \cdot 17^{3} \cdot 283}>1.21 \times 10^{-4}
$$

and

$\frac{2^{8} \cdot 3^{8} \cdot 17^{3}}{\operatorname{rãd}(283) \operatorname{rãd}\left(5^{11} \cdot 13^{2}\right) \operatorname{rãd}\left(2^{8} \cdot 3^{8} \cdot 17^{3}\right)}=\frac{2^{8} \cdot 3^{8} \cdot 17^{3}}{283 \cdot 5^{11} \cdot 13^{2} \cdot 2^{0} \cdot 3^{8} \cdot 17^{3}}=\frac{2^{8}}{283 \cdot 5^{11} \cdot 13^{2}}$

which is less than $1.096 \times 10^{-10}$. After examining those exceptional $a b c$ triples in [11, Tabel 1] having the ratio $c / \operatorname{rãd}(a b c)<1$, we may think of the chance to drop the power $1+\varepsilon$ from the right-hand side of the above difference analogue of $a b c$ conjecture. But this is not any conjecture based on a careful inspection but no more than guesswork at this moment. We cannot even judge if it is available to give analogous examples to $1+2^{m+2}\left(2^{m}-1\right)=$ $\left(2^{m+1}-1\right)^{2}$ and the Jastrzebowski-Spielman example for the difference setting or not, either.

Now let us return to the positive integer $x$ and write it in the form

$$
x=\prod_{i=1}^{k} p_{j_{i}}^{\operatorname{ord}_{j_{i}}(x)}
$$

where $\operatorname{ord}_{j_{i}}(x) \geq 1$ for all $1 \leq j_{1}<\cdots<j_{k}$. Then, letting $n \in \mathbb{N}$, we may define an analogue of the factorial polynomial by

$$
x^{\bar{n}}=\prod_{i=1}^{k}\left(p_{j_{i}} p_{j_{i}+1} \cdots p_{j_{i}+n-1}\right)^{\operatorname{ord}_{j_{i}}(x)},
$$

with $1^{\bar{n}}=1$. A difference analogue of Fermat's last theorem may then be stated as follows. No three positive integers $a, b$, and c satisfy the equation $a^{\bar{n}}+b^{\bar{n}}=c^{\bar{n}}$ for any integer value of $n$ greater than 2. Of course, we do not accept $3 \cdot 4+5 \cdot 6=6 \cdot 7$ as an example for $n=2$ since this reduces to $2+5=7$ (and moreover 4 and 6 are not primes). It is trivial that $a^{\overline{1}}+b^{\overline{1}}=c^{\overline{1}}$ has a solution $(a, b, c)$ consisting of 1 or prime numbers, when and only when it is the triple of 2 and an odd prime pair except for trivial $1+1=2$ and $1+2=3$ then. Thus the existence of infinitely many of such solutions would imply the existence of infinitely many twin primes, which is still an open question. For $n=2$, it seems difficult to determine such prime solutions $\left(p_{i}, p_{j}, p_{k}\right)$, that is, those satisfying $p_{i} p_{i+1}+p_{j} p_{j+1}=p_{k} p_{k+1}$. One finds at least the triple $\left(1,5,2^{2}\right)$ including a single prime with 1 and satisfying $1^{\overline{2}}+5^{\overline{2}}=\left(2^{2}\right)^{\overline{2}}$. In order to remind ourselves of the way of our shift, let us note that the triple $\left(1,11,3^{2}\right)$ is not such a solution for $n=2$, since $\left(3^{2}\right)^{\overline{2}}$ is not $(3 \cdot 4)^{2}$ but $(3 \cdot 5)^{2}$. Our shift step $\kappa$ is however allowed to be strictly greater than one or even to be a negative integer, so the triple represented as $\left(1,13,2^{2} \cdot 3^{2}\right)$ can be our solution for $n=2$ of shift -1 with $p_{0}=1$, that is, $1+13 \times 11=2^{2} \cdot 3^{2} \times 1^{2} \cdot 2^{2}$. In each case, there is however an artificial treatment of the number $p_{0}=1$, so that we are eager for a spontaneous solution at least for $n=2$ with some shift step $\kappa$.

In Section 3 we have implemented an approach by Shapiro and Sparer [32] in assuming that the polynomials in Theorem 3.4 are pairwise relatively prime. Extending this result to the case where polynomials in any vanishing sub-sum are relatively prime using the method of de Bondt [8] is left as an open question. In [8] one finds also a generalization of the DavenportStothers inequality. We also leave the problem of extending our results to polynomials or holomorphic functions of more than two complex variables as open questions. Some related 
discussions and good lists of references are found in Hu and Yang's monograph [23, Chapter 5] as well as [22, Section 3.2].

Last but not least, we mention Vaserstein's paper [41] where he proved a quantum $a b c$ theorem for polynomials over an algebraically closed number field. Theorem 3.1 corresponds to the " $h$-version" of [41, Theorem 1.1] with different notation and proof. Again, we leave finding " $(h, q)$-versions" with $q \neq 1$ for the remaining results in our paper as a future project.

Acknowledgements Thanks are due to the referee for valuable comments and suggestions, especially for the encouragement to make an attempt at conjecturing number-theoretic analogs to Theorems 3.1 and 4.1.

Funding Open access funding provided by University of Eastern Finland (UEF) including Kuopio University Hospital.

Open Access This article is licensed under a Creative Commons Attribution 4.0 International License, which permits use, sharing, adaptation, distribution and reproduction in any medium or format, as long as you give appropriate credit to the original author(s) and the source, provide a link to the Creative Commons licence, and indicate if changes were made. The images or other third party material in this article are included in the article's Creative Commons licence, unless indicated otherwise in a credit line to the material. If material is not included in the article's Creative Commons licence and your intended use is not permitted by statutory regulation or exceeds the permitted use, you will need to obtain permission directly from the copyright holder. To view a copy of this licence, visit http://creativecommons.org/licenses/by/4.0/.

\section{References}

1. Birch, B.J., Chowla, S., Hall Jr., Marshall, Schinzel, A.: On the difference $x^{3}-y^{2}$. Norske Vid. Selsk. Forh. (Trondheim) 38, 65-69 (1965)

2. Brownawell, W.D., Masser, D.W.: Vanishing sums in function fields. Math. Proc. Cambrid. Philos. Soc. 100(3), 427-434 (1986)

3. Cartan, H.: Sur la fonction de croissance attachée à une fonction méromorphe de deux variables et ses applications aux fonctions méromorphes d'une variable. C. R. Acad. Sci. Paris 189, 521-523 (1929)

4. Cartan, H.: Sur lés zeros des combinaisons linéaires de $p$ fonctions holomorphes données. Math. Cluj 7, 5-31 (1933)

5. Cherry, W., Toropu, C.: Generalized ABC theorems for non-Archimedean entire functions of several variables in arbitrary characteristic. Acta Arith. 136(4), 351-384 (2009)

6. Cherry, W., Ye, Z.: Nevanlinna's Theory of Value Distribution. Springer, Berlin (2001)

7. Davenport, H.: On $f^{3}(t)-g^{2}(t)$. Norske Vid. Selsk. Forh. (Trondheim) 38, 86-87 (1965)

8. de Bondt, M.: Another generalization of Mason's ABC-theorem. arXiv:0707.0434 (2007)

9. de Smit, B.: Abc triples. http://www.math.leidenuniv.nl/ desmit/abc/. Accessed 6 Jan 2020

10. Dyakonov, K.M.: Zeros of analytic functions, with or without multiplicities. Math. Ann. 352(3), 625-641 (2012)

11. Geuze, G., de Smit, B.: Count along with ABC. Nieuw Arch. Wiskd. (5) 8(1), 26-30 (2007)

12. Goldberg, A.A., Ostrovskii, I.V.: Value distribution of meromorphic functions, Translations of Mathematical Monographs, vol. 236. American Mathematical Society, Providence (2008) (Translated from the 1970 Russian original by Mikhail Ostrovskii. With an appendix by Alexandre Eremenko and James K, Langley)

13. Granville, A., Tucker, T.J.: It's as easy as $a b c$. Not. Am. Math. Soc. 49(10), 1224-1231 (2002)

14. Gross, F.: On the equation $f^{n}+g^{n}=1$, Bull. Am. Math. Soc. 72, 86-88 (1966). Correction: 72:576 (1966)

15. Gundersen, G.G., Hayman, W.K.: The strength of Cartan's version of Nevanlinna theory. Bull. Lond. Math. Soc. 36(4), 433-454 (2004)

16. Halburd, R.G., Korhonen, R.J., Tohge, K.: Holomorphic curves with shift-invariant hyperplane preimages. Trans. Am. Math. Soc. 366(8), 4267-4298 (2014)

17. Hayman, W.K.: Warings Problem für analytische Funktionen. Bayer. Akad. Wiss. Math. Natur. K1. Sitzungsber. 1984, 1-13 (1985)

18. Hayman, W.K.: Waring's theorem and the super Fermat problem for numbers and functions. Complex Var. Elliptic Equ. 59(1), 85-90 (2014) 
19. Hsia, L.-C., Wang, J.T.-Y.: The $A B C$ theorem for higher-dimensional function fields. Trans. Am. Math. Soc. 356(7), 2871-2887 (2004)

20. Hu, P.-C., Yang, C.-C.: The " $a b c$ " conjecture over function fields. Proc. Jpn. Acad. Ser. A Math. Sci. 76(7), 118-120 (2000)

21. Hu, P.-C., Yang, C.-C.: A note on the abc conjecture. Commun. Pure Appl. Math. 55(9), 1089-1103 (2002)

22. Hu, P.-C., Yang, C.-C.: Value Distribution Theory Related to Number Theory. Birkhäuser, Basel (2006)

23. Hu, P.-C., Yang, C.-C.: Distribution Theory of Algebraic Numbers, De Gruyter Expositions in Mathematics, vol. 45. Walter de Gruyter GmbH \& Co. KG, Berlin (2008)

24. Laeng, E.: Fermat's last theorem for polynomials. Parabola 35(1), 1-5 (1999)

25. Lang, S.: Old and new conjectured diophantine inequalities. Bull. Am. Math. Soc. (N.S.) 23(1), 37-75 (1990)

26. Li, N.: On the existence of solutions of a Fermat-type difference equation. Ann. Acad. Sci. Fenn. Math. 40(2), 907-921 (2015)

27. Mason, R.C.: Diophantine equations over function fields, London Mathematical Society Lecture Note Series, vol. 96. Cambridge University Press, Cambridge (1984)

28. Mason, R.C.: Norm form equations. I. J. Number Theory 22(2), 190-207 (1986)

29. Nevanlinna, R.: Analytic functions, Translated from the second German edition by Phillip Emig. In: Die Grundlehren der mathematischen Wissenschaften, Band 162, Springer, New York (1970)

30. Ozawa, M.: On the existence of prime periodic entire functions. Kōdai Math. Sem. Rep. 29(3), 308-321 (1977/1978)

31. Rubel, L.A.: Entire and Meromorphic Functions Universitext. Springer, New York (1996). (With the assistance of James E. Colliander)

32. Shapiro, H.N., Sparer, G.H.: Extension of a theorem of Mason. Commun. Pure Appl. Math. 47(5), 711-718 (1994)

33. Shioda, T.: Elliptic surfaces and Davenport-Stothers triples. Comment. Math. Univ. St. Pauli 54(1), 49-68 (2005)

34. Shioda, T.: The $a b c$-theorem, Davenport's inequality and elliptic surfaces. Proc. Jpn. Acad. Ser. A Math. Sci. 84(4), 51-56 (2008)

35. Snyder, N.: An alternate proof of Mason's theorem. Elem. Math. 55(3), 93-94 (2000)

36. Stewart, C.L., Tijdeman, R.: On the Oesterlé-Masser conjecture. Monatsh. Math. 102(3), 251-257 (1986)

37. Stothers, W.W.: Polynomial identities and Hauptmoduln. Q. J. Math. Oxf. Ser. (2) 32(127), 349-370 (1981)

38. Taylor, A.E.: L'hospital's rule. Am. Math. Mon. 59(1), 20-24 (1952)

39. van Frankenhuysen, M.: The ABC theorem for meromorphic functions (2008). arXiv: 0805.1729v1

40. van Frankenhuysen, M.: Hyperbolic spaces and the ABC conjecture. Ph.D. Thesis, Katholieke Universiteit Nijmegen (1995)

41. Vaserstein, L.N.: Quantum ( $a b c)$-theorems. J. Number Theory 81(2), 351-358 (2000)

42. Vojta, P.: Diophantine approximations and value distribution theory. In: Lecture Notes in Mathematics, vol 1239. Springer, Berlin (1987)

43. Voloch, J.F.: Diagonal equations over function fields. Bol. Soc. Brasil. Mat. 16(2), 29-39 (1985)

44. Woods, A.: Some problems in logic and number theory, and their connections, Ph.D. Thesis, University of Manchester (1981)

45. Zannier, U.: On Davenport's bound for the degree of $f^{3}-g^{2}$ and Riemann's existence theorem. Acta Arith. 71(2), 107-137 (1995)

46. Zannier, U.: Acknowledgement of priority Addenda: "On Davenport's bound for the degree of $f^{3}-g^{2}$ and Riemann's existence theorem”. Acta Arith. 74(4), 387 (1996)

Publisher's Note Springer Nature remains neutral with regard to jurisdictional claims in published maps and institutional affiliations. 